\title{
$Y$
}
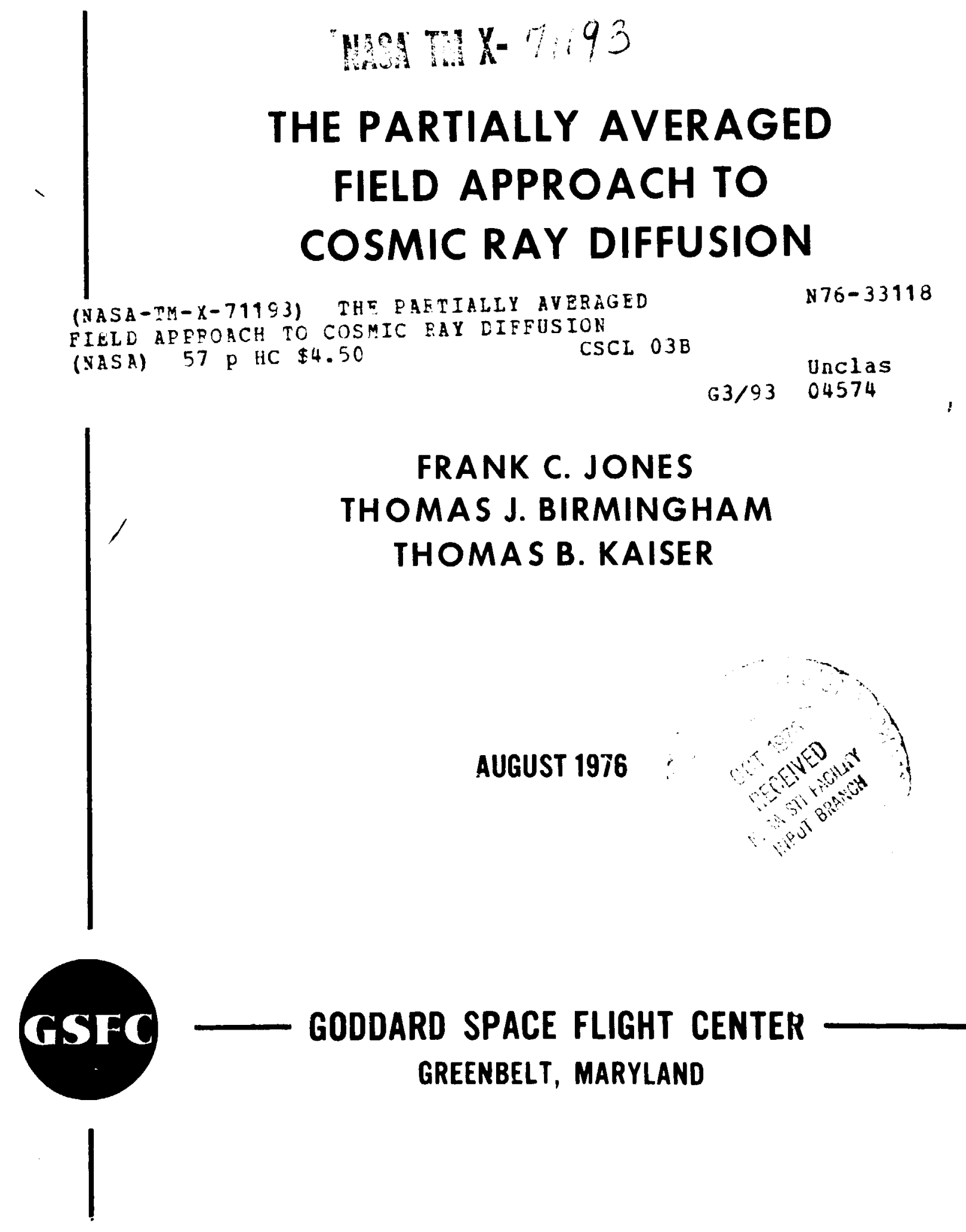

FRANK C. JONES

THOMAS J. BIRMINGHAM

THOMAS B. KAISER

AUGUST 1976 


\section{THE PARTIALLY AVERAGED FIELD APPROACH TO COSMIC RAY DIFFUSION}

Frank C. Jones Thomas J. Birmingham Thomas B. Kaiser*

August 1976

NAS-NRC Resident Reseorch Associate. Present Address:

Lawrence Livermore Laboratory, Livermore, CA.

\section{GODDARD SPACE FLIGHT CENTER}

Greenbelt, Maryland 20771 
Abstract......................... iii

I. Introduction. ....................... 1

II. Derivation of the Kinetic Equation. . . . . . . . . . . . 4

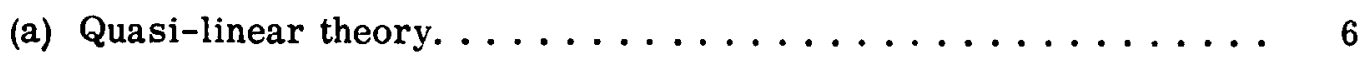

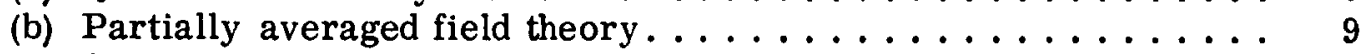

(c) Gaussian Statistics . . . . . . . . . . . . . . . . . 13

III. The Magnetic Slab Model . . . . . . . . . . . . . . . . . 13

(a) Results for the Perpendicular Slab Model . . . . . . . . . . . 18

(b) Interpretation of the Differences between PAF and

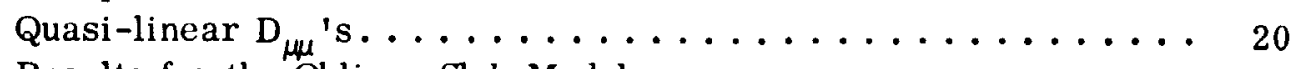

(c) Results for the Oblique $\mathrm{Slab}$ Model ................. 26

IV. Spatial Transport and the Scaling of $\mathrm{D}_{\mu \mu}^{\mathrm{PA}}(\mu \cong 0) \ldots \ldots \ldots \ldots \ldots$

V. Range of Validity of Partially Averaged Field Theory . . . . . . . . 32

APPENDIX A - Conditional Averaging for Multi-Dimensional Gaussian Processes. ................ 39

APPENDIX B - The Gyrotropy of $\langle\mathrm{f}\rangle \ldots \ldots \ldots \ldots \ldots$ 


\title{
THE PARTIALLY AVERAGED FIELD APPROACH TO COSMIC RAY DIFFUSION
}

\begin{abstract}
The kinetic equation for particles interacting with turbulent fluctuations is derived by a new non-linear techrique which successfully corrects the difficulties associated with quasi-linear theory. In this new method the effects of the fluctuations are evaluated along particle orbits which themselves include the effects of a statistically averaged subset of the possible configurations of the turbulence. The new method is illustrated by calculating the pitch angle diffusion coefficient $\mathrm{D}_{\mu \mu}$ for particles interacting with "slab model" magnetic turbulence, i.e., magnetic fluctuations linearly polarized transverse to a mean magnetic field $\langle\underset{\sim}{\mathrm{B}}\rangle$. Results are compared with those of quasj-linear theory and also with those of Monte Carlo calculations reported in a companion paper. The major effect of the non-linear treatment in this illustration is the determination of $D_{\mu \mu}$ in the vicinity of $90^{\circ}$ pitch angles where quasi-linear theory breaks down. The spatial diffusion coefficient $\kappa_{\|}$parallel to $\langle\underset{\sim}{\mathrm{B}}\rangle$ is evaluated using $\mathrm{D}_{\mu \mu}$ as calculated by our technique. It is argued that the partially averaged field method is not limited to small amplitude fluctuating fields and is hence not a perturbation theory.
\end{abstract}




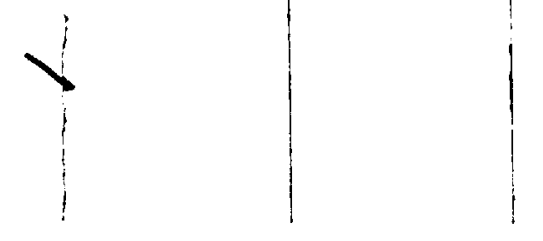

\section{THE PARTIALLY AVERAGED FIELD APPROACH TO COSMIC RAY DIFFUSION}

\section{Introduction}

The systematic treatment of the transport of charged particles in disordered magnetic fields began about ten years ago. ${ }^{1-3}$ These authors derived a FokkerPlanck equation for the time evolution of the particle distribution function by a method that is commonly referreci to as the quasi-linear method. ${ }^{4}$

A more formal definition of the quasi-linear approach will be given in the next section but the basic idea may be quickly sketched. To compute the mean square effect of a random force field on a particle one must be able to follow the particle's orbit in that field for such a time as is required for the random force to become self-incoherent. This is an inherently non-linear problem and in general intractable; however, if the random force can be separated into an average part, for which orbits can be calculated, and a random part that is small (in some sense) one may use the orbit in the average field to compute the effects of the random part in the hope that the random part will become self-incoheren, before it has a chance to significantly perturb the orbit.

There are two approaches one may take in applying these notions. One is to assume that the correct equation of evolution is a Fokker-Planck equation and proceed to calculate the appropriate coefficients. This approach has been developed primarily by Jokipii ${ }^{1,5}$ and by Hasselmann and Wibberenz. ${ }^{6}$ The difficulty in such an approach is the proper treatment of non-random forces.? The second method is to begin with the Vlasov equation and derive a Fokker-Planck 

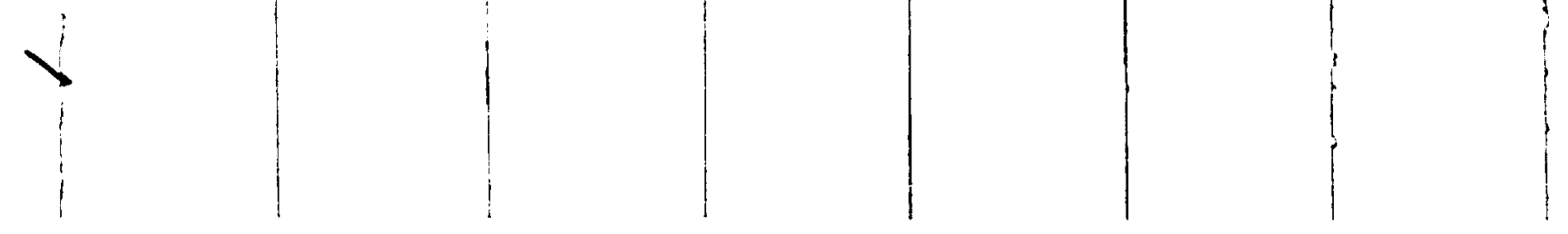

equation to describe the evolution of the ensemble averaged distribution function. The ensemble is the collection of all possible random fields with the required statistical parameters. This approach was employed by Hall and Sturrock, ${ }^{3}$ Roelof, ${ }^{2}$ Kulsrud and Pearce, ${ }^{8}$ and discussed in a general analysis by Kaufman. ${ }^{9}$ Most recent authors have employed this approach and we shall use it in this paper.

The first questions about the appropriateness of a Fokker-Planck equation for describing charged particle transport in random magnetic fields were raised by Klimas and Sandri. ${ }^{10}$ They pointed out that the presence of particle trajectories (in the average field) with pitch angles near or at $90^{\circ}$ with respect to the average field invalidated the "adiabatic hypothesis." Since this hypothesis is necessary in order to derive the Fokker-Planck equation from a non-Markovian integrodifferential equation resulting from previous steps in the derivation, these authors asserted that the correct transport equation was the more primitive (and more complicated) non-Markovian one.

The present authors then pointed out " that these same $90^{\circ}$ pitch angle particles caused even worse problems. Such particles remain in a region of correlated field for an arbitrarily long time and thus their orbit perturbations could become important before the random force becomes self-incoherent, violating the assumptions of quasi-linear theory. Indeed we were able to show that for a particular, simple model the terms that are dropped in quasi-linear theory eventually dominate for such particles.

Although the difficulty with quasi-linear theory is confined to a region of phase space that is usually small, this region about $90^{\circ}$ pitch angles can be quite important in considering the bulk transport of the charged particles. Earl ${ }^{12}$ has shown that if the fluctuations in the magnetic field are depleted at high frequencies 

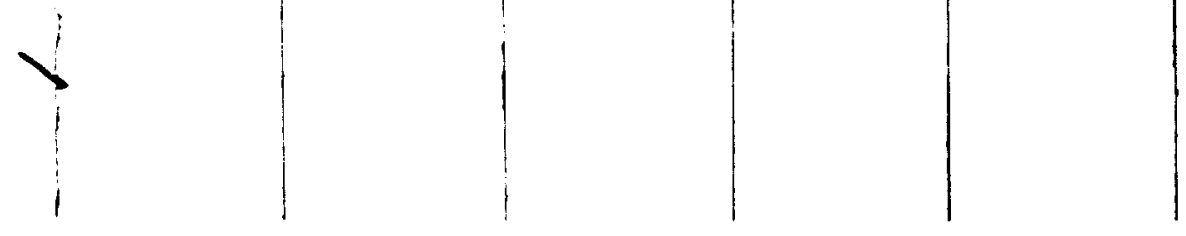

we proceed with generality. F has a component which fluctuates randomly in space and/or time. For our cosmic ray application of the theory $\underset{\sim}{F}$ is thought to be an "externally imposed" force, although the additional constraint of Maxwell's Equations can be added straightforwardly.

We describe the particles by their one partic'e distribution function $f(r, p, t)$ which, when the particles are interacting only with an externally imposed $\underset{\sim}{\mathrm{F}}$, obeys exactly the Vlasov Equation

$$
\left(\frac{\partial}{i t}+\frac{\mathrm{Q}}{\mathrm{m} r} \cdot \underset{\sim}{\nabla}+\underset{\sim}{\mathrm{F}} \cdot \frac{\partial}{\partial \underline{p}}\right) \mathrm{f}-0
$$

We are interested in the coarse-grained behavior of $\mathrm{f}$ - ite evolution on the time scale of several coherence times between the particles and the fluctuating force. We invoke the usual ergodic hypothesis and assert that this behavior is identically the behavior of ( $f$ ), which is $f$ averaged over an ensemble of $F_{r}^{\prime} s$. The ensemble of F's is specified by the values of the moments

$$
\begin{aligned}
& \langle\underline{\mathbf{F}}\rangle
\end{aligned}
$$

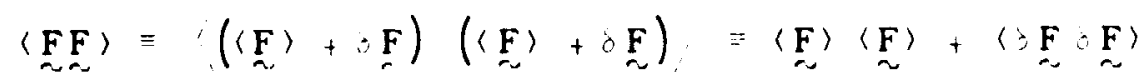

$$
\begin{aligned}
& \langle\underset{\sim}{\mathbf{F}} \underset{\sim}{\mathbf{F}}\rangle=\langle\underset{\sim}{\mathbf{F}}\rangle\langle\underset{\sim}{\mathbf{F}}\rangle\langle\underset{\sim}{\mathbf{F}}\rangle+\langle\underset{\sim}{\mathbf{F}}\rangle\langle\underset{\sim}{\mathbf{F}} \underset{\sim}{\mathbf{F}}\rangle+\langle\underset{\sim}{\mathbf{F}}\langle\underset{\sim}{\mathbf{F}}\rangle\langle\underset{\sim}{\mathbf{F}}\rangle+\langle\underset{\sim}{\mathbf{F}} \dot{\mathbf{F}}\rangle\langle\underset{\sim}{\mathbf{F}}\rangle \\
& +\langle\lesssim \mathbf{F} \circlearrowright \underset{\mathbf{F}}{\mathbf{F}}\rangle
\end{aligned}
$$

etc.

Equation (1) is readily split into an equation for if ?

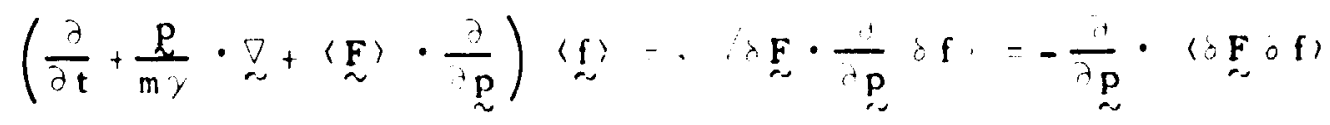



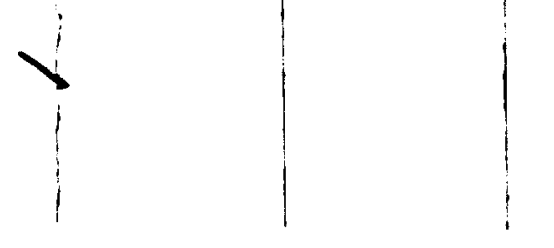

We discard the initial value term from further consideration on the assumption that after a reasonable interval $t-t_{0}$ all correlation between $F$ and $U_{0}\left(t, t_{0}\right)$ $\delta \mathbf{f}\left(\mathbf{r}, \underset{\sim}{\mathbf{p}}, \mathrm{t}_{0}\right)$ has died away so that ensemble averaging yields 0 . If such correlations do in fact persist, we think that it is impossible to describe (f) by the simple Markovian description toward which we strive. We assume that a similar argument applies when $U_{0}$ is replaced by the more correct propagator that we introduce later.

It. is the spirit of quasi-linear theory that scattering is small over a particlefluctuation coherence time. The evolution of $\langle f\rangle$ in Eq. $(5\rangle$ is predominantiy a convection of $(f)\left(t_{0}\right)$ along the characteristic trajectories in $(\underline{F}\rangle$. The quasilinear collision term

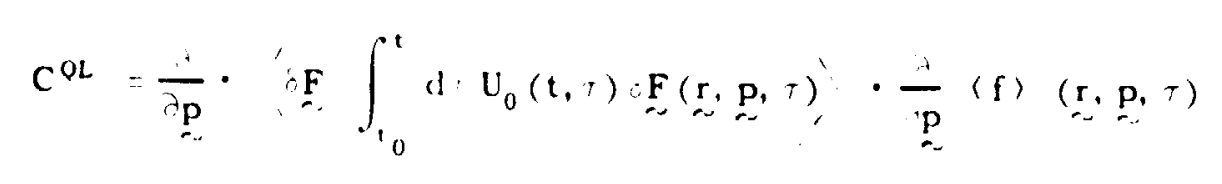

represents a small correction. It follows then that the adiabatic approximation $\mathrm{U}_{0}(\mathrm{t}, \tau)$ (f) $(\mathrm{r}, \underset{\sim}{\mathrm{p}}, \tau) \because(\mathrm{f})(\mathrm{r}, \underline{\mathrm{p}}, \mathrm{t})$ can and should be made in every instance that the quasi-linear theory is valid. ${ }^{18}$ The quasi-linear collision term is thus properly written

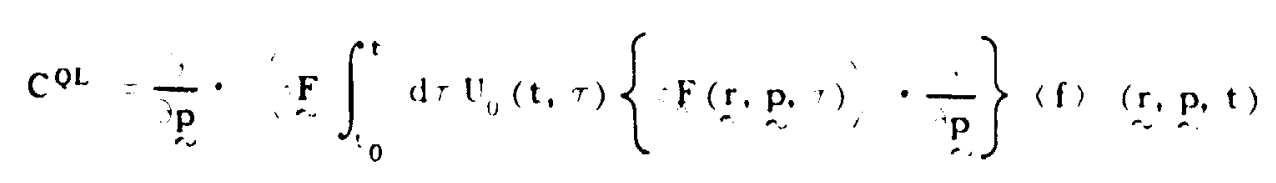

The brackets $;$ indicate that $\mathrm{U}_{0}$ now propagates $-\mathbf{F} \cdot / \mathrm{p}$ only.

We now examine the circumstances under which quasi-linear theory is valid, i.e. $\mathrm{C}^{\mathrm{OL}}$ is "small." It can be shown ${ }^{18}$ that quasi-linear theory is valid for most regions of the particle phase space provided that $\underbrace{E}_{\sim}$ and $B$ are small in the sense ' 'efined in Ref. 18). The reason is that the correlation $\left\langle\mathbf{F}(\mathbf{r}, p, t) U_{0}\left(t,{ }^{\prime}\right)\right.$ $F(r, p, r)\rangle$ diminishes rapidly with $t-T$. This decrease occurs in the 
The first term represents pure momentum diffurion. For times $t-t_{0} \gg t$ this diffusion is described by the tensor

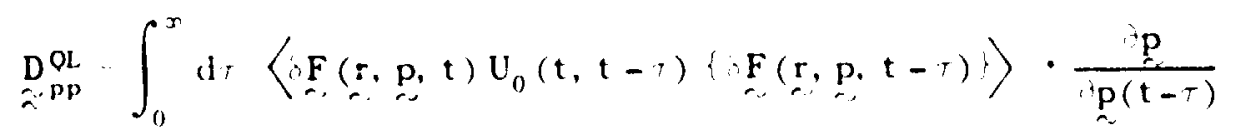

There is in addition a mixed $\underline{p}, \ddot{i}$ diffusion, which for large $t-t_{0}$ is described by the tensor

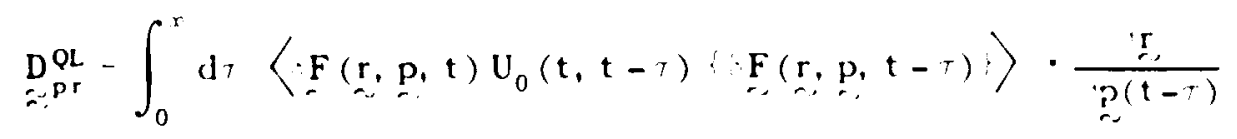

This mixed $\underset{\sim}{\mathrm{p}}, \underset{\sim}{\mathrm{r}}$ diffusion disappears when $\langle\mathrm{f}\rangle$ is spatially homogeneous and may be small for weak inhornogeneities. It is generally present, however, to the same degree as the pure momentum diffusion.

\section{(b) Partially averaged ficld theory}

In many models there are, however, pathological though important regions of phase space where $\tau_{i}^{(0)} \quad$ is quite long, so long in fact that $?_{i}^{1 .} \geq \tau_{s c a}$, and both the spirit and the substance of quasi-linear theory are iolated.

We propose here an improved approximation, which includes the essential effect of the fluctuations themselves in the lowest order propagator. For reasons that will be soon evident, we call our method the partially averaged field approximation. In this formalism the coherence time ${ }_{T}^{P A}$ is naturally limited $+{ }_{C}^{P A} \leq+$, and the collision integral is validly ca!culated for (1) all regions of the particle phase space and (2) arbitrary amplitude of the fluctuations. Our method is not a weak-coupling approximation. It reduces to quasi-linear theory when $\tau_{i}^{01} \ll T^{\prime}$ 《A holds. 


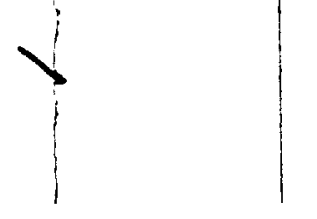

Our formalism proceeds as follows. We consider the ensemble averaging process to occur in two steps. A first partial average, denoted by \langle\rangle$_{c}$, is performed over all realizations with the same fixed values of $\delta \underset{\sim}{\mathrm{E}}(\mathbf{r}, \mathrm{t})$ and $\circ \mathrm{B}(\mathbf{r}, \mathrm{t})$ at the point $r$ and time $t$ at which Eq. (2) is valid. A second final average, indidated by \langle\rangle$_{i}$, is then carried out over the distributions of $\delta \underset{\sim}{\mathrm{E}}(\boldsymbol{r}, \mathrm{t})$ and $\underset{\sim}{\mathrm{B}}(\underset{r}{\mathrm{r}}, \mathrm{t})$. The collision term in Eq. (2) is identically written according to this prescription

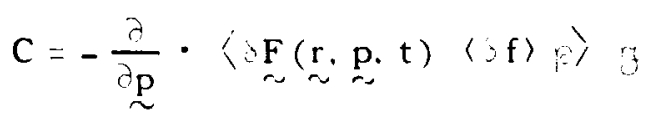

If $\mathrm{f}(\mathrm{r}, \mathrm{p}, \mathrm{t})$ depended only on $\mathrm{F}(\mathrm{r}, \mathrm{p}, \mathrm{t})$ it would be the same for every realization included in the partial ensemble and partial averaging would be trivial. However, $\{\mathbf{f}$ depends on $\mathbf{F}$ globally. We therefore write Eq. (3) for the arbitrary point $\underline{r}^{\prime}, \underline{\sim}^{\prime}, t^{\prime}$ and partially average to obtain

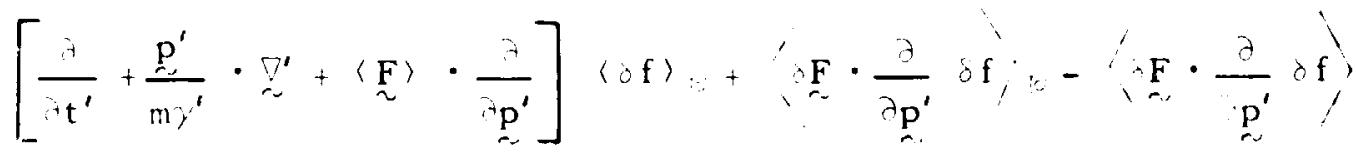

$$
\begin{aligned}
& =-\langle S F\rangle \cdot \frac{\langle f\rangle}{\partial p^{\prime}}
\end{aligned}
$$

Note that partially averaged quantities depend functionally on $\underset{\sim}{r^{\prime}}, \underline{p}^{\prime}$, and $t^{\prime}$ which are the variables of Eq. (11) and parametrically on $\underset{r}{r}$ and $t$ which are singled out by the partial averaging procedure. Thus far Eqs. (10) and (11) are exact, for all we have done is to break the full ensemble average procedure into two sequential steps: symbolically $(),\langle\langle\rangle\rangle$

The essence of our method is to approximate in Eq. (11) as follows

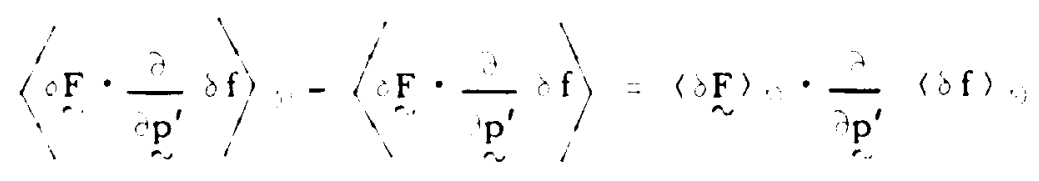




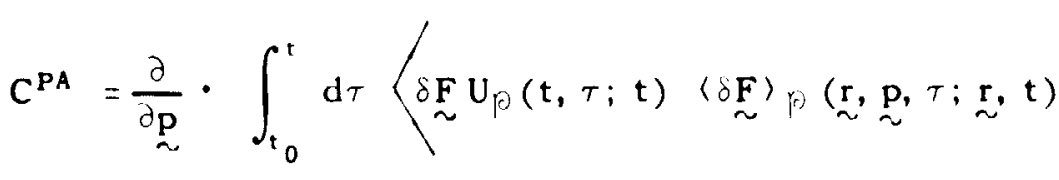

$$
\begin{aligned}
& \left.\frac{\partial\langle\boldsymbol{f}\rangle(\mathbf{r}, \mathbf{p}, \tau)}{\partial \underline{p}}\right\rangle
\end{aligned}
$$

We expect decay of $\left.\left\langle\delta \underset{\sim}{\mathrm{F}} \mathrm{U}_{j}(\mathrm{t}, \tau ; \mathrm{t})\langle\delta \underset{\sim}{\mathrm{F}}\rangle_{\mathrm{p}} \underset{\sim}{\mathbf{r}} \underset{\sim}{\mathrm{p}}, \tau ; \underset{\sim}{\mathbf{r}}, \mathrm{t}\right)\right\rangle$ in the time $\mathrm{t}-\tau=\mathrm{t}_{\mathrm{C}}^{\mathrm{PA}}$ $\left(t_{c}^{P A} \leq t_{c}^{Q L}\right)$ sufficiently short that all characteristic orbits in the partially averaged force remain at least marginally valid for the entire time interval over which the integrand in (15) is non-zero. We hold this expectation irrespective of the size of $\delta \mathrm{F}$ and shall argue why in Section $\mathrm{V}$.

For times $\mathrm{t}-\mathrm{t}_{0} \gg \tau_{\mathrm{C}}^{\mathrm{PA}}$ we again write

$$
\begin{aligned}
& C^{P A}=\frac{\partial}{\partial p} \cdot \int_{0}^{D} \mathrm{~d} \tau\left\langle\delta F U_{W}(t, t-\tau ; t)\langle\delta F\rangle(r, p, t-\tau ; r t r) \cdot\right. \\
& \left.\frac{\partial\langle\mathbf{f}\rangle(\underline{q}, \underline{p}, \mathbf{t}-\tau)}{\partial \underline{p}}\right\rangle
\end{aligned}
$$

The adiabatic approximation on $\langle f\rangle$ cannot always be made in $\mathrm{C}^{\mathrm{PA}}$. The physical reason is that when $\delta F$ is substantial, scattering as described by Eq. (16) represents a significant correction to the convective motion in $\langle\underset{\sim}{F}\rangle$. Our success in deriving an equation unrestricted by the size of $\delta \underset{-}{\mathrm{F}}$ is thus somewhat mitigated by the fact that this equation for $\langle f\rangle$ is an integro-differential equation. One must not conclude, however, that an adiabatic approximation is never possible: each model to which the (f) equation is applied must be examined individually to see whether an adiabatic ansatz can be made consistently with the resulting physics. Work reported in later sections is illustrative of this point. 


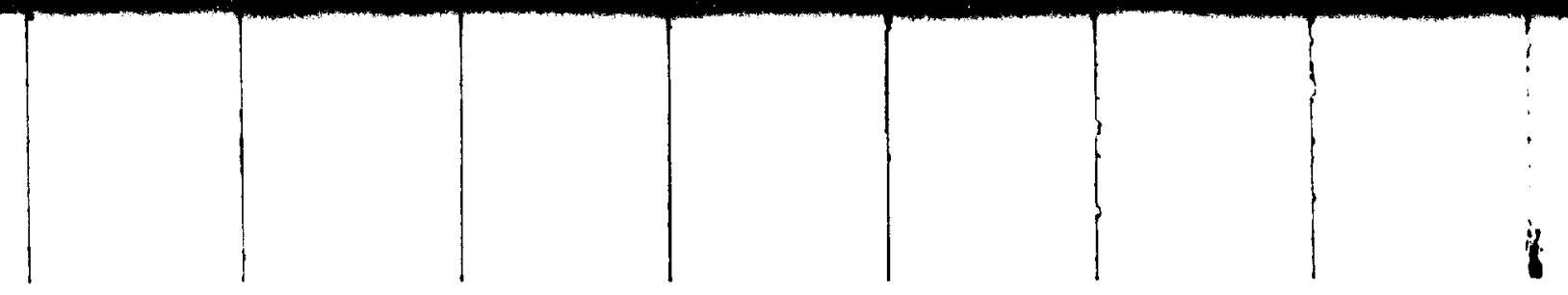

\section{(c) Gaussian statistics}

It is impossible to proceed further with the partially averaged field method in full generality. We always consider the fluctuating electric and magnetic fields, $\delta \underset{\sim}{\mathbf{E}}(\underline{r}, t)$ and $\delta \underset{\sim}{\mathbf{B}}(\underline{\mathbf{r}}, \mathrm{t})$, to be Gaussian processes. It can be shown (Appendix A) for a Gaussian process that

$$
\begin{aligned}
& (\delta \underset{\sim}{E})_{\gamma}\left(\stackrel{\sim}{\prime}^{\prime}, t^{\prime} ; \underset{\sim}{r}, t\right)=\delta \underset{\sim}{E}(\underset{\sim}{r}, t) \cdot \underset{\approx}{C_{2}}\left(\underset{\sim}{r^{\prime}}, t^{\prime}, \underset{\sim}{r}, t\right)
\end{aligned}
$$

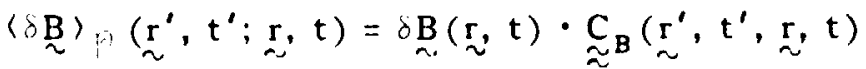

where $\underset{\approx}{\mathrm{C}}$ and $\underset{\approx B}{\mathrm{C}}$ are the normalized correlation tensors for the fluctuating electric and magnetic fields respectively.

The assumption of a Gaussian process is a common one, partly because the central limit theorem ${ }^{19}$ states that a tendency towards Gaussian statistics is a common occurrence in random systems, but mostly because of its mathematical tractability which most other types of statistics do not share. Mathematically, Eqs. (17) follow uniquely - and $\delta \mathrm{E}$ and $\mathrm{B}$ are Gaussian processes - provided that one demands that $\langle\delta \underset{\sim}{\mathbf{E}}\rangle,\left(\mathbf{r}^{\prime}, \mathbf{t}^{\prime} ; \underset{\sim}{\mathbf{r}}, \mathbf{t}\right)$ and $\langle\delta \underset{\sim}{\mathrm{B}}\rangle\left\langle\left(\mathbf{r}^{\prime}, \mathbf{t}^{\prime} ; \underset{\sim}{\mathbf{r}}, \mathbf{t}\right)\right.$ be linearly dependent respectively on $\delta \underset{\sim}{\mathrm{E}}(\underset{\sim}{\mathbf{r}}, \mathrm{t})$ and $\delta \underset{\sim}{\mathrm{B}}(\underset{\sim}{\mathbf{r}}, \mathrm{t})$.

\section{The Magnetic Slab Model}

We have worked out the details of the partially averaged field formalism for the so-called magnetic slab model, and we shall discuss details in this section. The magnetic slab model was chosen because its simplicity facilitates calculation and direct comparison with Monte Carlo results (which are reported in the accompanying paper ${ }^{20}$ ) and because it also contains some of the basic physics of the scattering of cosmic rays from irregularities in the interplanetary medium. 
In the magnetic slab model there are no electric fields. The ensemble average magnetic field $\langle\underline{-}\rangle\rangle$ is spatially homogeneous and oriented along the $z$-axis of a Cartesian coordinate system; magnetic fluctuations are linearly polarized, constant in time, and vary in magnitude only with distance along $\langle\underline{B}\rangle$. Thus $\delta \mathbf{B}=\delta(\mathrm{B}) \hat{\mathrm{e}}_{\mathbf{x}}$. In this simplest form, there is no spatial variation in planar slabs oriented perpendicular to the $\mathrm{z}$-axis and hence the coinage "slab model." At the end of this section we discuss a more generalized slab model in which $\mathrm{B}$ is in the $\widehat{\mathrm{e}_{\mathrm{x}}}$ direction but planes of constant $|0 \mathrm{~B}|$ are 1 to the $\mathrm{y}-\mathrm{z}$ plane and at a fixed angle (typically $30^{\circ}$ ) with respect to the z-axis.

The magnetic slab model is intended as a description of the interaction of cosmic rays with slow magnetic structures (tangential discontinuities or Alfven waves) in the solar wind. Let us assume that the radial divergence of the solar wind flow can be neglected and that we can place ourselves therefore in a unique solar wind frame of reference. The amplitude of the electric fields associated. with these slow structures is at most $|E|=v_{A} \mid B ; / c$ and hence far smaller than the magnitude of the magnetic fields $\left(v_{A}\right.$ is the Alfven speed and is typically 50$100 \mathrm{~km} / \mathrm{sec}$ ). The magnetic force on relativistic cosmic rays is therefore far larger than the electric force, and for that reason we neglect the latter.

Our assumption that $\langle\underset{\sim}{\mathrm{B}}\rangle$ is completely uniform breaks down, of course, on the $1 \mathrm{AU}$ spatial scale of the classical Archimedes spiral. However, the pertinent scale for a description of scattering is the autocorrelation distance of magnetic fluctuatione, which has been measured to be a few hundredths of an $\mathrm{AU} ;{ }^{21}$ to the extent that $(B)$ is uniform over this length our model is a good one. There is also evidence 22 that the inagnetic fluctuations are preferentially polarized in the direction normal to the ecliptic plane (the normal direction would thus correspond 


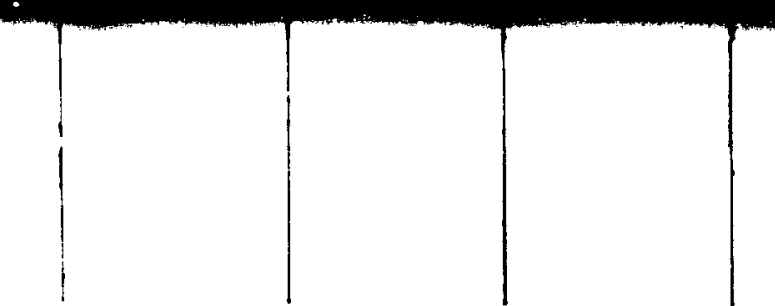

to our $\mathrm{x}$-direction); any component of $\underset{\sim}{\mathrm{B}}$ in the ecliptic plane and normal to ( $\mathrm{B}$ ) at its point of generation gradually develops a compressional component ${ }^{23}$ as it propagates outward in the solar wind and tends to damp away. ${ }^{24}$

Since electric fields are negligible, cosmic rays suffer no energy changes in their interactions with the fluctuations and $|p|$ is simply a parameter in the equation for $(\mathrm{f}\rangle$. Scattering in angle does occur and can be described in terms of the variables $\mu=\mathrm{p} \cdot \hat{\mathrm{e}}_{z} /|\mathrm{p}|$ and $\phi=\tan ^{-1} \mathrm{p}_{\mathrm{x}} / \mathrm{p}_{\mathrm{y}} \cdot \mu$ is the cosine of the cosmic ray's pitch angle relative to $\langle\underset{\sim}{\mathrm{B}}\rangle$ and $t$ is the gyrophase angle measured from $\mathrm{B}$ (the $\mathrm{x}$-axis).

We consider here the situation where $(f)$ is spatially homogeneous. Such a situation arises when $\langle f\rangle$ is homogeneous at $t=t_{0}$ and the spectrum of the fluctuations is itself spatially homogeneous. This assumption of spatial homogeneity is artificial and unrealistic: as a result of it the bulk spatial transport of cosmic rays along and across field lines is precluded. It is made solely to achieve a simple model in which to explore and justify the partially averaged field method. The spatially inhomogeneous situation will be discussed in Section IV.

With the aforementioned approximations the equation for $\langle f\rangle$ is

$$
\begin{aligned}
& \frac{i\langle f\rangle}{\lambda t}-\langle,\rangle \frac{\langle f\rangle}{\lambda t}-D(f, t) d \int_{t}^{r} d+U(t, t-\tau) j \\
& \mathrm{D}(i, t)\langle\mathrm{f}\rangle\langle\mathrm{r}, \mathrm{p}, \mathrm{t}-\tau)\rangle
\end{aligned}
$$

In Eq. $(18),()=,\mathrm{q}(\mathrm{B}) /, \mathrm{mc}$ is the gyrofrequency in the ensemble average magnetic field $(\mathrm{B}) \widehat{\mathrm{e}}_{\boldsymbol{z}}, \partial_{\alpha}$ is the gyrofrequency $\mathrm{q} / \mathrm{B} / / \gamma \mathrm{mc}$ that would prevail if $\cdot:_{\sim}^{B} \widehat{e}_{x}$ were the only magnetic field, and $D$ is the differential operator 


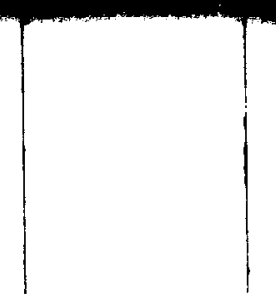

$$
\begin{aligned}
\mathrm{D}= & (1-\mu)^{1 / 2} \sin \phi \frac{\partial}{\partial \mu}-\frac{\mu \cos \phi}{\left(1-\mu^{2}\right)^{1 / 2}} \frac{\partial}{\partial \phi}= \\
& \frac{\partial}{\partial \mu}\left(1-\mu^{2}\right)^{1 / 2} \sin \phi-\frac{\partial}{\partial \phi} \frac{\mu \cos \phi}{\left(1-\mu^{2}\right)^{1 / 2}}
\end{aligned}
$$

We next approximate further by neglecting the gyrophase dependence of $\langle f\rangle$. This is accomplished formally by first expanding (f) in the Fourier series

$$
\langle f\rangle=\sum_{n=-\infty}^{+\infty}\langle f\rangle_{n} \exp \text { in } \phi \text {. }
$$

next substituting into Eq. (18) and projecting out the $n=0$ component

$$
\begin{aligned}
& \frac{\partial\langle\mathrm{f}\rangle}{\partial t} 0=\frac{1}{2 \pi} \int_{0}^{2 \pi} \mathrm{d} \cdot \frac{\partial}{\partial \mu}\left(1-\mu^{2}\right)^{1 / 2} \sin t\left\langle\int_{0}^{x} d+U_{i j}(t, t-\tau ; t)\right. \\
& \operatorname{sic}\left[\left(1-\mu^{2}\right)^{1 / 2} \sin +\frac{\partial}{\partial \mu}-\frac{\mu \cos }{\left(1-\mu^{2}\right)^{1 / 2}}-\frac{\partial}{\mu}\right] \\
& \left.\sum_{n=-\infty}^{+\infty x}\langle f\rangle_{n} \exp i n\right\rangle
\end{aligned}
$$

and finally neglecting the $n \neq 0$ terms on the right hand side of Eq. (21). Fourier amplitudes $\left.\langle f\rangle_{n}, n\right\rangle 0$, are small compared to $\langle f\rangle_{0}$ in the approximate ratio $\eta^{2}=\left\langle\partial \mathrm{B}^{2}\right\rangle /\langle\mathrm{B}\rangle^{2}$ (cf. Appendix B) provided that they are initially small; the physical reason being that the convective term $(w)(f) / \lambda \neq$ keeps particles well stirred in $t$ Thus 


$$
\begin{aligned}
& \frac{\partial\langle\mathrm{f}\rangle}{\partial t}=\frac{1}{2 \pi} \int_{0}^{2 \pi} d ; \frac{\partial}{\partial \mu}\left(1-\mu^{2}\right)^{1 / 2} \sin \left\langle\left\langle\omega \int_{0}^{x} \mathrm{~d} \tau \mathrm{U}_{\psi}(\mathrm{t}, \mathrm{t}-\tau ; \mathrm{t})\right.\right. \\
& \left.\partial\left(1-\mu^{2}\right)^{1 / 2} \sin t \frac{\partial\langle f\rangle_{0}}{\partial \mu}(\mu, \tau)\right\rangle
\end{aligned}
$$

Next note

$$
\dot{\mu}=\frac{\dot{p}_{2}}{p}=-\frac{\delta \omega p_{y}}{p}=-\omega\left(1-\mu^{2}\right)^{1 / 2} \sin \phi
$$

so that

$$
\frac{\partial(\mathrm{f})_{0}}{\partial \mathrm{t}} \frac{1}{\mu} \frac{1}{2 \pi} \int_{0}^{2 \pi} \mathrm{d} \phi\left\langle\dot{\mu} \int_{0}^{\infty} d \tau \mathrm{U}, \dot{\mu} \frac{\partial(\mathrm{f})_{0}(\mu, \tau)}{\partial \mu}\right\rangle
$$

To the right de of Eq. (23) must be added any effects such as sinks and sources of particles which are not included in the original Vlasov description Eq. (1).

In the steady state $(f\rangle_{0}$ no longer depends explicitly on time and (23) can therefore be written in the more transparent form

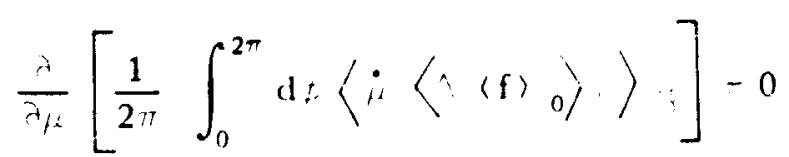

Here

$$
\left\langle\Delta\langle f\rangle_{0}\right\rangle=\int_{0}^{x} d \tau U_{i} ; \frac{t(f)_{0}(k)}{d \tau}-\int_{i ;} d+\frac{d(f)_{0}}{d \tau}
$$

is the change in ( $f)_{0}$ apparent to an observer moving from the point $\mu$ at $t=0$ along an orbit in the partially averaged field $(\stackrel{\sim}{\mathbf{B}})$.

To the extent that $(f\rangle_{0}(\mu)$ is a linear function of $\mu_{\mu}$ - and we have found from our Monte Carlo calculations that such is approximately the case for a wide variety of conditions - 


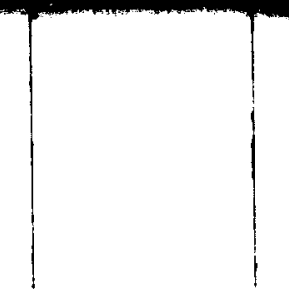

$$
\left\langle\Delta\langle f\rangle_{0}\right\rangle_{\tilde{O}} \cong\langle\Delta \mu\rangle_{i} \frac{\partial\langle f\rangle_{0}}{\partial \mu}
$$

and Eq. (24) is of diffusion form

$$
\frac{\partial}{\partial \mu}\left(\mathrm{D}_{\mu \mu}^{\mathrm{PA}} \frac{\partial\langle\boldsymbol{f}\rangle_{0}}{\partial \mu}\right)=\frac{\partial}{\partial \mu}\left[\frac{1}{2 \pi} \int_{0}^{2 \pi} \mathrm{d} \phi\langle\dot{\mu}\langle\Delta \mu\rangle,\rangle_{i} \frac{\partial\langle\boldsymbol{f}\rangle_{0}}{\partial \mu}\right]=0
$$

The diffusion coefficient $D_{\mu \mu}^{P A}$ has exactly the same form as the quasi-linear diffusion coefficient $D_{\mu \mu}^{\mathrm{QL}}$ but differs in that $\Delta_{\mu}$ is the change in $\mu$ evaluated following an orbit in the partially averaged field rather than following a helical orbit in $(\underset{\sim}{B}\rangle$ as quasi-linear theory prescribes. For a $/=0$ particle in the slab model $\Lambda_{1}$ approaches no finite limit as $\tau \rightarrow \propto$ in quasi-linear theory ${ }^{25}$ because such a particle suffers exactly canceling deflections over its circular orbit at constant $\mathbf{z}$ in $\langle\underline{B}\rangle$; no such pathological behavior occurs in the partially averaged field method because even $\mu=0$ particles move freely in $\mathrm{z}$ and hence enter regions of uncorrelated $s \mathrm{~B}$.

(a) Results for the Perpendicular Slab Model

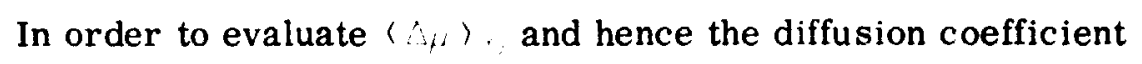

$$
\left.\mathrm{D}_{1 \mu \mu}^{P A}-\frac{1}{2 \pi} \int_{0}^{2 \pi} \mathrm{d}:\langle i\langle\hat{i}\rangle\rangle\right\rangle
$$

Newton's Equations of motion

$$
\begin{aligned}
\frac{d p^{\prime}}{d \tau}=\underline{p}^{\prime} \times\left[(,) \widehat{c}_{z}+\therefore(z) C\left(z-z^{\prime}\right) \widehat{e_{x}}\right] & \\
& \frac{d \underline{r}^{\prime}}{d \tau}-\frac{p^{\prime}}{y^{\prime} m}
\end{aligned}
$$


are integrated numerically backward in time from the boundary conditions $\underline{\sim}^{\prime}(\tau=t)=\underset{\sim}{p}, \underline{\sim}^{\prime}(\tau=t)=\underset{\sim}{r}$. The correlation function $C$ is chosen to be the exponential

$$
C\left(z-z^{\prime}\right):=\frac{1}{z_{c}} \exp -\frac{\left|z-z^{\prime}\right|}{z_{c}}
$$

since we compare $\mathrm{D}_{\mu \mu}^{\mathrm{PA}}$ with the results of a Monte Carlo simulation, ${ }^{20}$ for which it is simplest to generate numerically magnetic fields with an exponential correlation function. The correlation distance $z_{c}$ is the distance scale and the correlation time $\mathbf{t}_{\mathrm{c}}=\mathrm{z}_{\mathrm{c}}, \mathrm{m} / \mathrm{p}$ the unit of time.

We first pick a $(z)$ and the boundary values $\mu(\tau=\mathrm{t})$ and $t(\tau=\mathrm{t})$. is for inclusion in (26) follows immediately from Eq. $(22) .\left\langle\Delta_{\mu}\right\rangle$, , on the other hand is the change in $/ 2$ which results from the integration of Eqs. (27). This integration is carried out until the particle has moved typically a distance $z^{\prime}-z \simeq 16 z_{c}$ in the direction of varying $\left(z^{\prime}\right)$.

The entire procedure is next averaged over a uniform distribution of $:(t)$. The density with which the :(t) must be chosen depends on .. (t). When the latter is small $\left(f_{-}(\mathrm{t}) \leq \therefore,(\mathrm{z}) /(),\right)$ particle mirroring is an important effect, as we shall see shortly. For small,$\ldots$, the $y^{\prime}$ are taken at $1^{\circ}$ intervals in order to achieve convel'gcuce for the :-averaging process. For larger,$:(\geq,,(z) /()$,$) mirroring$ dees not occur and it is sufficient to take :(t)'s which are $2^{\circ}$ apart.

The last step in the evaluation of $D_{\mu L L}^{P A}$ is the final averaging procedure, in which we perform the steps of the preceding two paragraphs for several different values of $:(z)$, typically 20 , each weighted by the distribution

$$
P(,) \frac{1}{\frac{2+\left(a^{2}\right)}{2 !(2)}} \exp -\frac{2^{2}}{2\left(2^{2}\right)}
$$




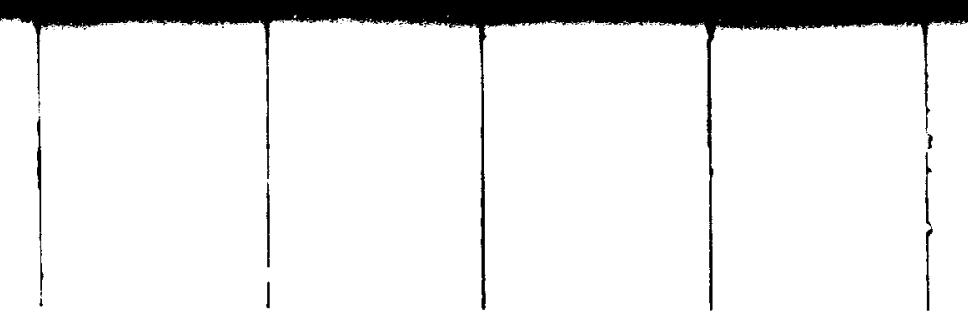

Figures 1 to 3 depict $D_{\mu \mu}^{P A}$ thus computed as a function of $\mu$. Each figure corresponds to particular values of $\eta_{l}=\left\langle\partial^{2}\right\rangle 1 / 2 /(\alpha)$ and $+=y \mathrm{~m}(n) \mathrm{z}_{\mathrm{c}} / \mathrm{p}$. The discrete points at which $D_{\mu \mu}^{P A}$ is calculated are indicated. The solid curve is a spline fit to these points. Shown also on each graph is the curve of $D_{i \mu \mu}^{\mathrm{QL}} \mathrm{vs}$ $\mu$. This quasi-linear diffusion coefficient is computed from the formula

$$
\mathrm{D}_{\mu L}^{\mathrm{QL}} \quad-\left.\frac{1-\mu^{2}}{2}\right|_{\mu} \mid \frac{\eta^{2}}{\epsilon^{2}+\mu^{2}}
$$

which results when the exponential correlation function (28) is assumed.

Equation (29) is the limiting $\tau \cdots$ value of the expression

$$
\left\langle i(\mathrm{t}) \wedge, \mathrm{OL}^{\mathrm{O}} \quad(\mathrm{t}-\mathrm{t})\right\rangle
$$

wherever that limit exists. As we have mentioned earlier and demonstrated elsewhere ${ }^{25}$ this limit does not exist as $\ell^{\prime} \rightarrow 0$ and indeed a quasi-linear diffusion coefficient is not properly defined at such small in's.

We would point out three aspects of these figures: (1) the peaking and definitely non-zero value of $D_{\mu \mu}^{\mathrm{PA}}$ as $\mu \rightarrow 0,(2)$ the fact that $\mathrm{D}_{\mu \mu}^{\mathrm{PA}}$ is approximately equal to but slightly less than $D_{\mu \mu}^{\text {QI. }} \quad$ for .. $\because, \quad$ and (3) the essential equality of $D_{\mu \mu}^{P A}$ and $D_{\mu \mu}^{\mathrm{OL}}$ as $\mu \rightarrow 1$. These aspects are also characteristic of the diffusion coefficient $D_{\mu \mu}$ obtained from Monte Carlo computer experiments. 20

(b) Interpretation of the Differences between PAF and Quasi-Linear $\mathrm{D}_{t .:}$ 's.

We interpret the bump which appears in the vicinity of $\ldots=0$ as due to particle mirroring: particles with pitch angles near $90^{\circ}$ are dominantly affected by the maximum in $\langle\mathrm{B}\rangle$, at $\mathrm{z}=0$ and their , 's are determined by magnetic moment conservation to be 


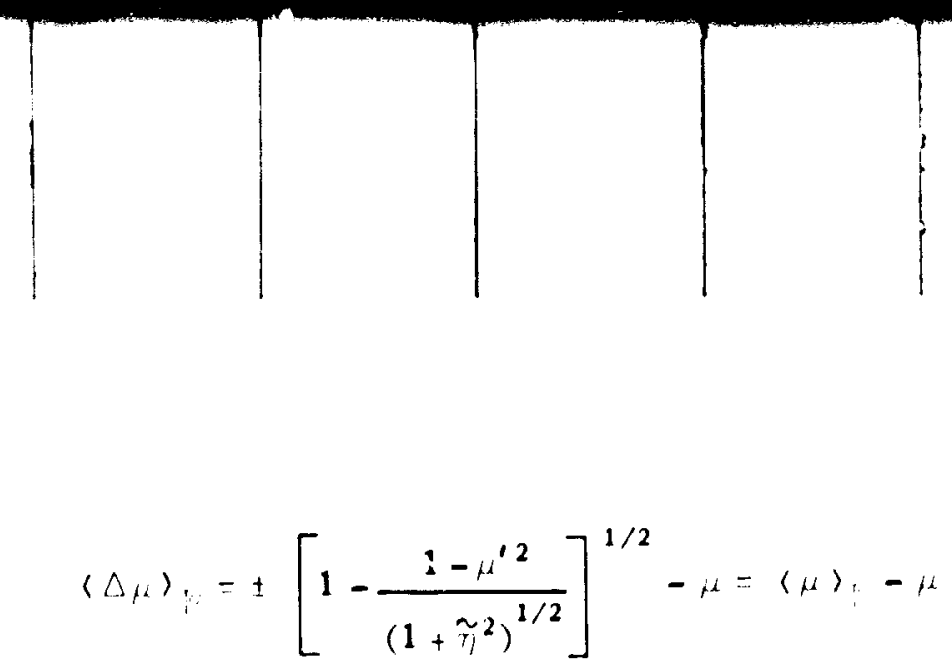

(Without loss of generality we assume here that $z=0$ is the point at which the diffusion coefficient is to be evaluated.) Here $\tilde{\eta}=\hat{b}(0) /\langle B\rangle$ and ${ }^{\prime}$ ' is the cosine of the particle's pitch angle with respc t to the total (partially averaged) field $\langle\underset{\sim}{B}\rangle$ :

$$
\mu^{\prime}=\frac{\mu}{\left(1+\tilde{\eta}^{2}\right)^{1 / 2}}+\frac{\left(1-\mu^{2}\right)^{1 / 2} \tilde{\eta_{l}}}{\left(1+\tilde{\eta}^{2}\right)^{1 / 2}} \cos
$$

Using Fq. (30) for $\Delta_{\beta}$ and Eq. (22) for $i$, we determine the mirroring contribution to $D_{\mu \mu}^{P A}$ thus to be (cf. Eq. (26))

$$
D_{\mu \mu}^{P A}-\frac{\left(1-\mu^{2}\right)^{1 / 2}}{2 \pi}\left\langle\int_{0}^{2 \pi} d i \sin :\left\{-\left[1-\frac{1}{\left(1+\mu_{i}^{2}\right)^{1 / 2}}\right]^{1 / 2}\right\}\right.
$$

The $\mp$ sign is included within the integral sign because the algebraic sign of $\langle\mu\rangle$ is dependent on $; ;$ if it were not, (32) would clearly integrate to zero because $\mu^{\prime}$ depends only on $\cos t$, and there would be no contribution of mirroring to the diffusion coefficient. Furthermore the sign cannot be just a function of $\cos :$ (as is the sign of $\mu^{\prime}$ ) or the same reasoning gives a zero result once again. We shall see that it is just the act of mirroring that injects the needed asymmetry about : $=0$, i.e., a dependence on $\sin ;$, to produce the peak of $\mu=0$.

To determine the sign of $\langle\mu\rangle$, we proceed in the following heuristic fashion. Consider the,$>0$ (but $\left.\right|_{i:} \mid \simeq 0$ ) particle at $\mathrm{z}=0$ at $\mathrm{t}=0$ in the partially averaged field. Depending on its : and $\mu$ this particle's guiding center is at positive or negative $\%$ 


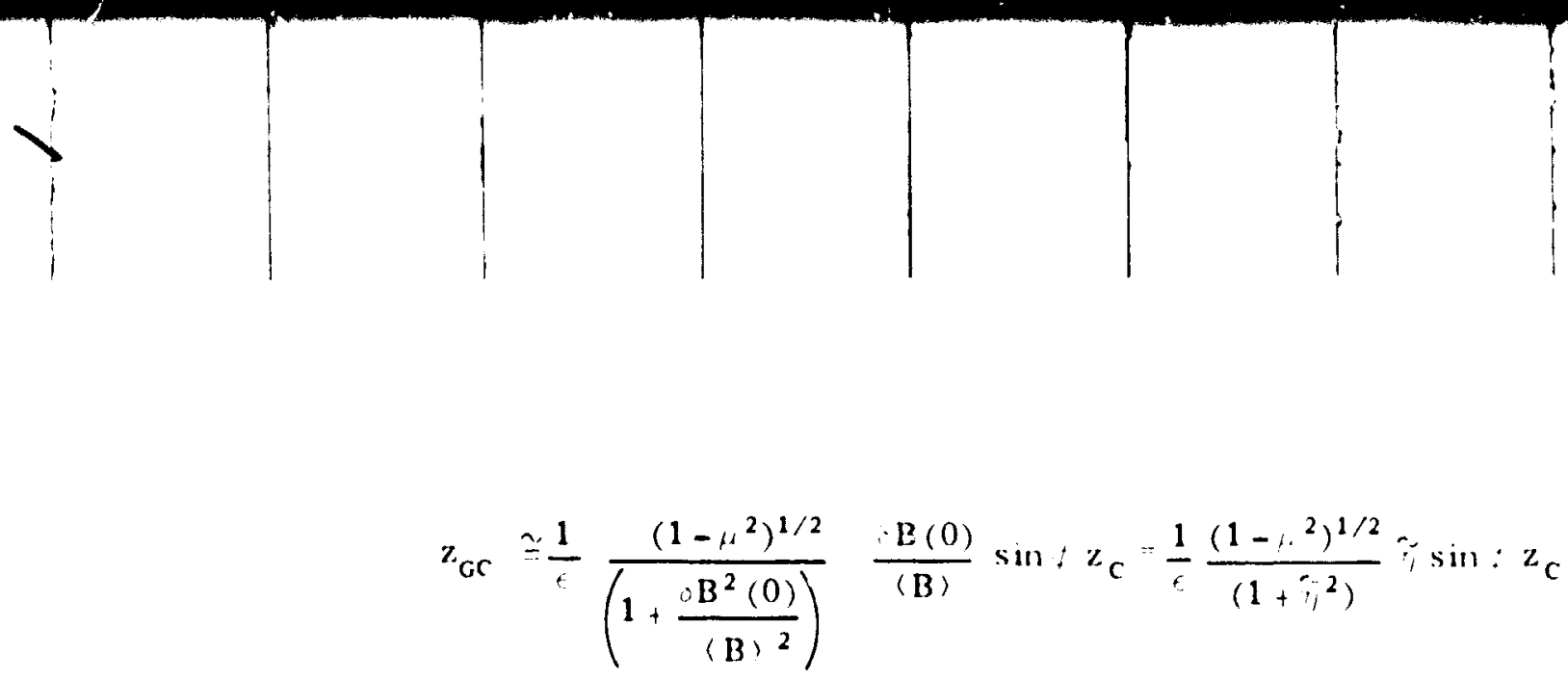

and its $\mu^{\circ}$ (cf. Eq. (31) $)$ is also positive or negative. If $\mathrm{z}_{\mathrm{GC}}$ and,$\Rightarrow$ are both $>0$, its initial motion carries the particle to the right (to larger $z$ ) and we assert that

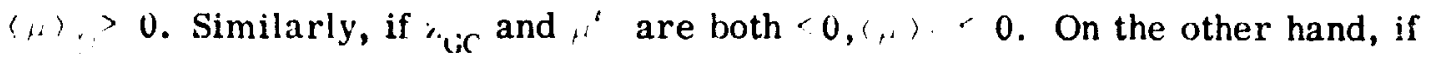
$Z_{G C}$ and ${ }^{\prime}$ ' have opposite algebraic signs, the particle's initial motion carries its guiding center toward $z=0$ and stronger magnetic field so that mirroring is a possibility. If mirroring does occur - and we shall presently develop criteria for mirroring $-(.$.$) . has the same sign as z_{G C}$. If mirroring does not occur, (..) , has the same sign as $: i^{\prime}$. In Figure 4, which depicts the transverse $p_{x}, p_{y}$ plane, mirroring may occur for $\dddot{i}>0$ in those sectors denoted by an "M?". (Because of the symmetry of the distribution of : $B(0)$, we may restrict our consideration to $\because>0$.) The sign of $(.$,$) . in the complementary sectors is also$ indicated. The angle $A$ is given by

$$
A-\sin ^{-1}\left[\frac{1}{\left(1-n^{2}\right)^{1 / 2}} \frac{1}{r}\right]
$$

When does mirroring in fact occur? We first applied the criterion that predicts mirroring when

$$
\frac{1-.^{2}}{(B) \cdot\left({ }^{2} \mathrm{GC}\right)}>\frac{1}{(\mathrm{~B}) \cdot(\mathrm{z}-0)} .
$$

On using the approximation

$$
\text { (B) }\left(z_{C C}\right)=(B) \quad(z=0)\left(1-\frac{z_{G C}}{z_{C}} \frac{\ddots^{2}}{1+r^{2}}\right)
$$


plus the expressions (31) and (33) for $\mu$ and ${ }^{\mathrm{G}} \mathrm{GC}$, the criterion for mirroring is equivalently written

$$
\left|\mu+\left(1-\mu^{2}\right)^{1 / 2} i \cos :\right|^{2}+\frac{r^{3}}{\epsilon} \frac{\left(1-\mu^{2}\right)^{2 / 2}}{\left(1+\eta^{2}\right)} \mid \text { sin }
$$

The :-integration in Fq. (32) has been performed numerically. The ambiguity in the sign of $\langle$,$\rangle , in the regions of Figure 4 r$ rked by "M?" is resolved by testing for the validity of (35) and assigning (..), the sign of $z_{G C}$ if (35) is indeed satisfied.

Following the procedure just prescribed, we have calculated the mirroring contribution to $\mathrm{D}_{\mu \mu}^{\mathrm{PA}}$ and fourd it in the vicinity of,$=0$ to be typically a factor of 2 larger than the $D_{\mu l i}^{P A}$ determined by exact integration of orbits in the partially averaged magnetic field: mirroring has been introduced too strongly in our heuristic procedure. Much better agreement is obtained when we modify the mirroring requirement (34) as follows:

$$
\frac{1-,: 2}{(B) \cdot(\% \mathrm{GC})}>\frac{1}{(\mathrm{~B}) \cdot(\mathrm{z}: 0)\left[1-\frac{\left(1-4^{2}\right)^{1 / 2} \cdot 3^{3}}{\left(1+i^{2}\right)^{3 / 2}} \frac{2}{\pi}\right]}
$$

The additional factor in the denominator of the right side accounts for the fact that even when the guiding center is located at $z=0$ the particle itself is almost always at a weaker field strength, owing to the $\exp -\left(\% / z_{C}\right)$ fall-off in $B_{x}$.

We attribute the fact that $\mathrm{D}_{L, .}^{P A}<\mathrm{D}_{L, L}^{\mathrm{OL}}$ in the regions.. $>$ of Figs. 1-3 to another effect, which we shall try to explain heuristically in this section. In quasi-linear theory, a particle moves with the assumed constant momentum $p$. in the $z$-direction. As time increases from $t=0$, the particle interacts in the 
Equation (39) differs from its quasi-linear analogue only in the fact that the oscillatory component of the $z$-motion is included, $\bar{\eta}$ in $(38)$ has been approximated by $\eta$ and the $\eta$ contribution to the rectilinear motion has been discarded.

It is now straightforward to calculate, using Eq. (26), the resonance contribution to $\mathrm{D}_{\mu \mu}$. We use (22) for $\hat{i},(39)$ for $\wedge_{\mu}$, assume no correlation between different Fourier modes (the random phase approximation), and employ the expansion

$$
\exp (-\mathrm{i} i 2 \sin \therefore)=\sum_{m=-a}^{+\infty} \exp -\mathrm{imi} \mathrm{J}_{\mathrm{m}}(\alpha)
$$

where the $J_{m}$ 's are the ordinary Bessel Functions. The result obtained for the exponential correlation function Eq. (28) is

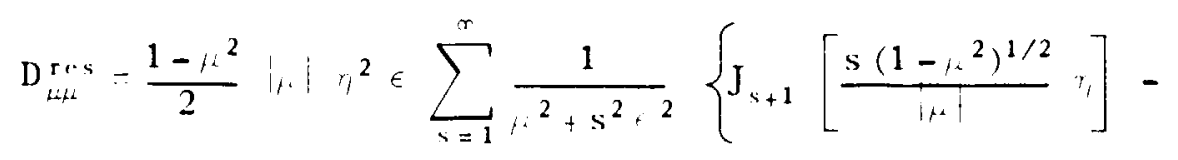

$$
\begin{aligned}
& \left.\mathrm{J}_{\mathrm{s}-1}\left[\frac{\mathrm{s}\left(1-\mu^{2}\right)^{1 / 2}}{|\mu|}, 1\right]\right\}^{2}
\end{aligned}
$$

The quasi-linear result is recovered by letting ,, 0 inside of the summation, only the $s=1$ term survives and Eq. (29) readily follows.

It is not obvious from Eq. (40) that the resonance interruption results in a diminished diffusion coefficient. We have, however, evaluated numerically as a function of ", the ratio

$$
\left(h^{2}+t^{2}\right) \sum_{s=1}^{m} \frac{1}{h^{2}+s^{2} t^{2}}\left\{J_{s+1}\left[\frac{s\left(1-\mu^{2}\right)^{1 / 2}}{|\mu|} \eta-J_{s-1}\left[\frac{s\left(1-\mu^{2}\right)^{1 / 2}}{|\mu|} \eta\right]\right\}^{2}\right.
$$



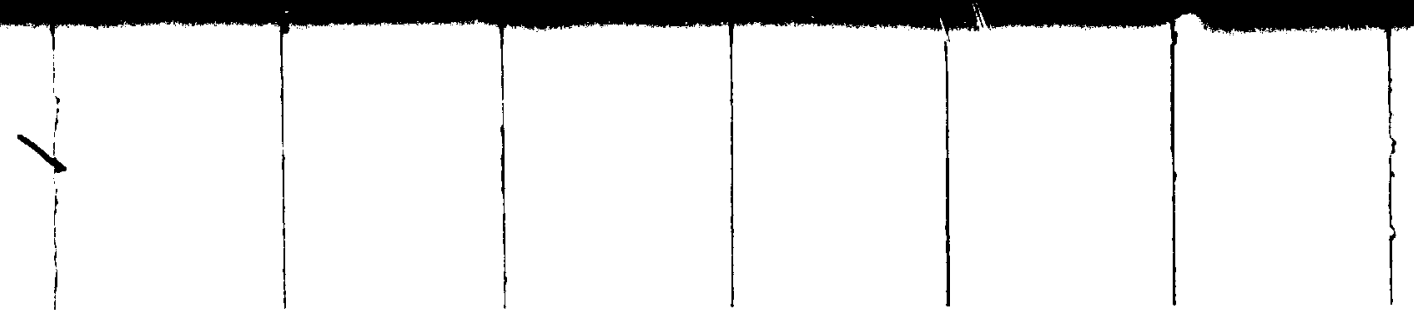

for several values of $\|$ and $\epsilon$. Its value is indeed $\leq 1$ and its general behavior is quite similar to that of $\mathrm{D}_{\mu \mu}^{\mathrm{P}} / \mathrm{D}_{\mu \mu}^{2 \mathrm{~L}}$ in Figs. $1-3$ in the ${ }_{\mu}>{ }_{\mu}$ range. This finding is the basis of our interpretation that resonance interruption is the reason that $D_{\mu \mu}^{P A}$ falls beneath $D_{\mu \mu}^{\mathrm{QL}}$ in this range.

(c) Results for the Oblique Slab Model

We have also evaluated $D_{\mu \mu}^{P A}$ using Eqs. (22), (26), and (27) for the situation where

$$
\langle B\rangle=b B(s) C\left(s-s^{\prime}\right) \hat{e}_{x}
$$

The correlation $\mathrm{C}$ is again exponential (cf. Eq. (28)) but in this instance

$$
\mathrm{s}-z \cos \alpha+y \sin \alpha
$$

so that planes of constant $\mid$ B $\mid$, are perpendicular to the $y-z$ plane and inclined at angle $r$ with respect to the $\mathrm{z}$-axis. (The perpendicular slab model considered in IIIa corresponds to $x=0$ ). Since the final averaging is over an assumed spatially homogeneous distribution of $\delta B^{2}(s)$, the position $s$ is again irrelevant and we may without loss of generality in (41) take $z=y=s=0$. The processes of averaging numerically over : and $\lrcorner B(s)$ are exactly as detailed in IIla.

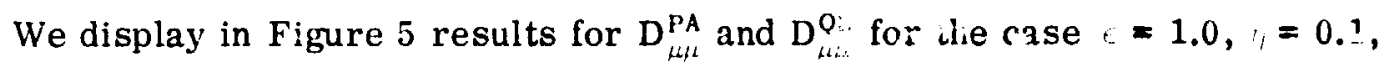
and $a=30^{\circ}$. Figure 5 is thus the counterpart of Figure 2 for the oblique aituation. The obvious difference between the oblique and pirndicular models is the strong suppression of diffusion at small $\mu$ 's which non-perpendicularity introduces. This is true for both $D_{\mu \mu}^{P A}$ and $D_{\mu \mu}^{(1)}$. In additiol: 1 -perpendicularity apparently leads to enhancements of $\mathrm{D}_{\mu \mu}$ in the region of maximum around $\mu=0.5$. The discrete values of $\mu$ for which $D_{\mu \mu}$ 's were explicitly evaluated are indicated in Fig. 5. The 
distribution and number of such points is dictated by the length of the numerics involved in calculating $\mathrm{D}_{\mu \mu}^{\mathrm{PA}}$. For comparison we have also calculated $\mathrm{D}_{\mu, \mu}^{\mathrm{QL}}$ at the same $\mu$ values. It is probable that the relatively small number of points in the region $\mu \cong 0$ leads to the suppression of small amplitude oscillations which characterize the higher harmonic (of $\langle$,$\rangle ) interaction of particles and magnetic per-$ turbations with spatial structure perpendicular to $\langle\mathrm{B}\rangle$. It is known that such oscillations characterize $\mathrm{D}_{\mu \mu}^{\mathrm{QL} .}{ }^{26}$ and it is probable that they would also appear if $D_{\mu \mu}^{P A}$ were calculated sufficiently densely in $\mu$. Our attitude is that if these oscillations have the same amplitude in $\mathrm{D}_{\mu \mu}^{\mathrm{PA}}$ as they have in $\mathrm{D}_{\mu \mu}^{\mathrm{QL}}$ they are inconsequential by comparison with the magnitude of $\mathrm{D}_{\mu \mu}^{\mathrm{PA}}(\mu \cong 0)$.

As we shall see in Section IV, the spatial diffusion coefficient $\kappa_{\|}$parallel to (B) varies inversely with $D_{\mu \mu}$. The important inference to be gained therefore from Fig. 5 is that the oblique slab situation is more conducive to parallel spatial i.ansport than is the perpendicular slab. Extrapolating to the case of the interplanetary medium, we expect that quasi-planar magnetic structures propagating radially outward from the sun undergo transition from a perpendicular slab-like situation to the oblique slab case as the idealized (B) develops spirally. ${ }^{22}$ Given constant : and $c, \kappa_{k}$ should therefore ideally decrease with approaching distance to the sun.

IV. Spatial Transport and the Scaling of $D_{\mu \mu}^{\mathrm{PA}}(\mu \simeq 0)$

Let us assume that pitch angle difínsion theory is true locally and that $D_{\mu \mu}$ is properly given by partially averaged fie:d theory. We re-introduce a spatial gradient in the $\mathrm{z}$-direction with scale much longer than $\mathrm{z}_{\mathrm{c}}$, and suppressing the suffix " 0 " rewrite Eq. (23) as 


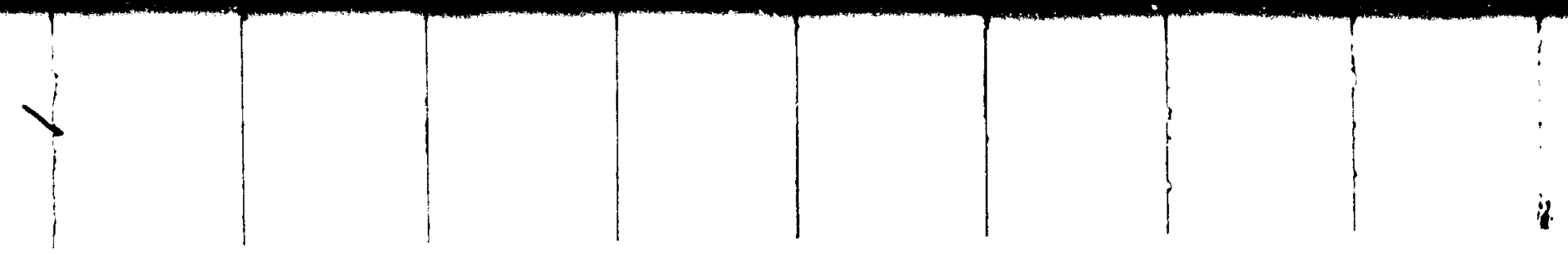

$$
\frac{\partial\langle\mathbf{f}\rangle}{\partial \mathbf{t}}+\mu \mathbf{v} \frac{\partial\langle\mathbf{f}\rangle}{\partial \mathbf{z}}=\frac{\partial}{\partial \mu} \mathrm{D}_{\mu \mu}^{\mathbf{P A}}(\mathbf{z}, \mathbf{v}, \mu) \frac{\partial\langle\mathbf{f}\rangle}{\partial \mu}
$$

Contact with the macroscopic variables - density $n$, flux $\Phi$ etc. - is made by taking moments of Eq. (42) with respect to $\mu$. Particle conservation

$$
\begin{aligned}
\frac{\partial n}{\partial t} & =-\int_{-1}^{+1} d \mu \mu v \frac{\partial\langle f\rangle}{\partial z}=-v \frac{\partial}{\partial z} \int_{-1}^{+1} \mathrm{~d} \mu \mu\langle f\rangle \\
& =-v \frac{\partial}{\partial z} \Phi
\end{aligned}
$$

follows excctly by taking the zeroth moment and noting that there can be no diffusive flux $D_{\mu \mu}^{P A} \partial\left\langle C^{\circ}\right\rangle / \partial \mu$ at the boundaries $\mu= \pm 1$ in $\mu$ space.

There exist a variety of methods for expressing $\Phi \equiv \int_{-1}^{1} d_{\mu \mu}\langle\mathbf{f}\rangle$ in terms of $\mathrm{n}$ and thus obtaining a closed equation for $\mathrm{n}$. The standard transport theory method ${ }^{27}$ is to expand $\langle f\rangle$ in Legendre Polynomials and truncate the series after $P_{1}(\mu)$. This yields a standard diffusion equation for $n$

$$
\frac{\partial n}{\partial t}=\frac{\partial}{\partial z} \kappa_{\|}^{L} \frac{\partial n}{\partial z}
$$

where

$$
\kappa_{\|}^{L}=\frac{\frac{2}{9} v^{2}}{\int_{-1}^{1} \mathrm{~d} \mu \mathrm{D}_{\mu \mu}(\mu)}
$$

Alternatively one could expand $\langle f\rangle$ in eigenfunctions of the diffusion operator $\lambda / \| D_{\mu \mu} d / \mu$ and obtain an alternate expression for $\kappa_{\|}^{E}{ }^{28}$ :

$$
k_{11}^{E}=\sum_{i=0 d i} \tau_{i} V_{0 i}^{2}
$$




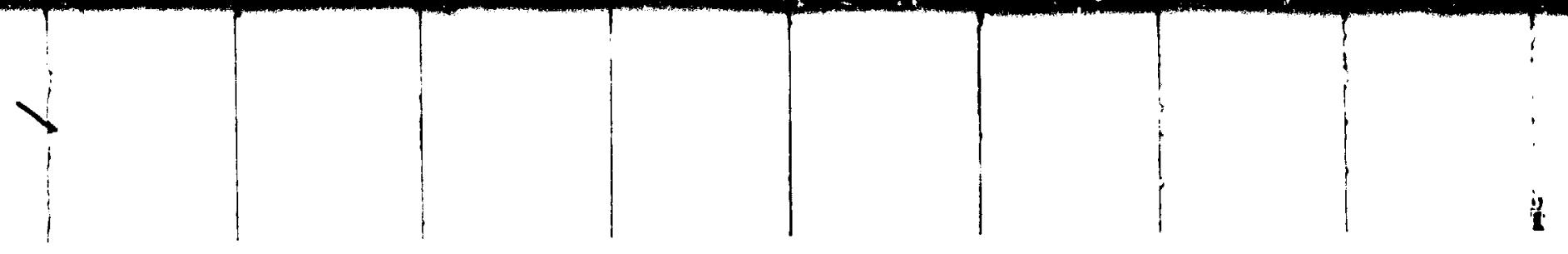

Earl $^{12}$ has compared the three approaches extensively and has concluded that the third one is the correct approach to employ. While we hesitate to call any approximation scheme "correct," we do concur that the third method has many arguments in its favor and is the result of a fully systematic approximation scheme.

However, it is known that if $\mathrm{D}_{\mu \mu}(\mu) \alpha\left(1-\mu^{2}\right)$ representing isotropic scattering, all three methods lead to the identical value for ${ }^{\star} \|$. Therefore if the form of $\mathrm{D}_{\mu \mu}(\mu)$ is not too different from that of isotropic scattering one would not expect it to matter very much which approach was used and convenience should dictate, especially in the field of astrophysics where the quantitative input is usually of a somewhat approximate nature.

We have computed values of $\kappa_{\|}$by both the Legendra expansion method $\left(\kappa_{\|}^{L}\right)$ and the third or inversion method $\left(\kappa_{\|}^{J}\right)$ for the four sets of parameters for which we have complete curves of $\mathrm{D}_{\mu \mu}^{\mathrm{PA}}(\mu)$. The results are shown in Table 1.

As would be expected, the differences are most pronounced where $D_{\mu \mu}^{P A}(. .=0)$ is the smallest. The case $t=1,:=.1$ for the perpendicular slab may also be compared with the results of Goldstein ${ }^{29}$ who calculated $D_{\mu \mu}$ by an independent non-linear method ${ }^{15}$ and used Eqs. (45) and (51) to calculate $\kappa \frac{L}{\|}$ and $\kappa J$ (Goldstein uses the symbol $\kappa^{D}$ rather than our $\kappa_{\|}^{J}$.) Goldstein obtains $\kappa_{\|}^{L}=115$ and $\kappa_{\|}^{J}=165$. The near identity of ${ }_{\| l}^{L}$ with our results reflects the insensitivity of $<_{\|}^{L}$ (cf. Eq. 45) to $D_{\mu \mu}(=0)$, while his larger $\kappa_{\|}^{J}$ is the result of the fact that his non-linear method

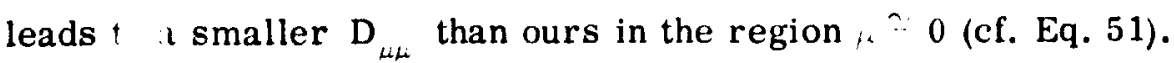

Sir: " $" \|$ depends inversely on the magnitude of $D_{\mu \mu}$ a know'ledge of how' $D_{\mu \mu t}$ scales with the parameters , and " would be of use in estimating the way " varies with the power in the fluctuating fields and particle rigidity. Since 


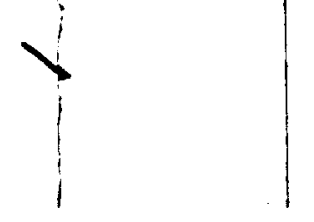

$\kappa_{11}$ depends on the shape of $\mathrm{D}_{\mu \mu}$ as a function of $\mu$ as well as the overall magnitude a simple scaling will not tell the whole story. However, since the value of $\kappa_{\|}$in Eq. (51) depends rather sensitively on the value of $D_{\mu \mu}$ at $\mu=0$ we have chosen this value as the scaling quantity of interest. We have therefore computed $\mathrm{D}_{\mu \mu}^{\mathrm{PA}}(\mu=0)$ for the nine different cases resulting from the combination of the $3 \epsilon-$ values $.25,1$, and 4 with the $3 \eta$-values $.05, .1$, and .3 , and we have attempted from these 9 cases to fit $D_{\mu \mu}^{P A}(\mu=0)$ to the form

$$
\mathrm{D}_{\mu \mu}^{\mathrm{PA}}\left(\mu_{i}=0\right) \sim \eta^{\mathrm{m}} \in \mathrm{U}
$$

For each value of $\epsilon$ we determined that $\mathrm{m}$ which gave best fit to the theoretically determined values for the 3 's. The linearity of $\log \left[D_{i \mu}^{\mathrm{PA}}(\mu<=0)\right]$ vs $\log \gamma_{/}$is excellent for each value of $\epsilon$. The optimum $\mathrm{m}$-values are $2.24,2.30$, and 2.34 for the cases $=.25,1$ and 4 respectively.

Similarly we determined $q^{\prime}$ 's holding each of the 3 values of fixed. The linearity of $\log \left[D_{\mu \mu}^{\mathrm{PA}}(\mu=0)\right.$ vs $\log \epsilon$ at constant $\eta$ is again excellent. The optimum q-values a.e $.58\left(\gamma_{l}=.05\right), .60\left(y_{1}=.1\right)$ and $.64\left(y_{l}=.3\right)$. Combining fits we propose the empirical scaling

$$
\mathrm{D}_{\mu \mu}^{\mathrm{PA}}(/ i=0) \sim r_{t}^{2.3} \epsilon^{.6}
$$

A similar effort to scale the values of $\mathrm{D}_{\mu, L}$ obtained from the Monte Carlo simulations described in the companion paper yields ${ }^{20}$

$$
D_{\mu \mu}^{M C} \quad(:-0) \sim 2.7, \cdot 3
$$

It is difficult to say whether the difference between the indices in Eqs. (52) and (53) is significant. But it is interesting to note that if one adopts the heuristic, 
where $D_{\mu \mu}$ is the pitch angle diffusion coefficient calculated by the theory in question. In this case the deflection time is given by

$$
\tau_{D}-\left(D_{\mu \mu}\right)^{-1} \text { for } \dot{o r}_{\mu} \ll(1)
$$

Clearly the random force must become incoherent in a time short compared to $\tau_{D}$ but there is another time of interest. The deflection time is a measure of how rapidly the particle orbit becomes unreliable in velocity space but the orbit can also become unreliable in coordinate space (in this model the $\mathrm{z}$ coordinate is the only one that matters). If $\mu$ is uncertain by an amount $s / \mu(t)$ the $z$ conrdinate will be uncertain by an amount

$$
\begin{aligned}
& z=v \int_{0}^{t} \cdots\left(t^{\prime}\right) d t^{\prime} \\
& =\frac{1}{2} v \cdots t^{2} \text { for } \therefore \lambda\left\langle t^{\prime}\right\rangle
\end{aligned}
$$

or

$$
-v t a d\langle\text { for } a, k(1)
$$

If this uncertainty in coordinate space grow's to be of the order of the correlation length $z_{\mathrm{c}}$ which is the characteristic scale of variation of the random field, the unperturbed orbit becomes quite useless. The time in which this occurs is called the deviation time and is given by

$$
\begin{aligned}
& \tau_{d}=\left(\frac{z_{c}}{v a_{d}}\right)^{1 / 2} \text { for } c_{1} \geq(d) \\
& =\frac{x_{c}(\cdot)}{v_{b}} \text { for } x \ll\langle(,) \text {. }
\end{aligned}
$$



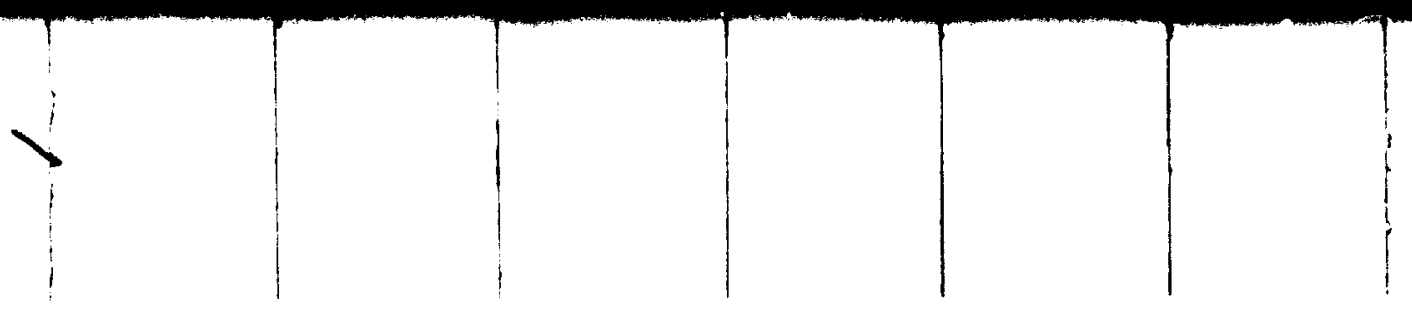

We further require that this deviation time be long compared to the time required for the random force to become self-incoherent.

Just as we have defined two time scales over which the approximate particle orbit becomes unreliable we may define two coherence time scales, one for configuration space and one for velocity space. This is possible because the random force $F=(q / c) v \times \delta B(z)$ depends on both $z$ and $v$. Clearly the shorter of these two times is the time over which $\mathrm{F}$ becomes incoherent.

The correlation length $z_{c}$ is defined as the length scale over which the random field $\mathrm{B}$ becomes self-incoherent, it is therefore natural to define the correlation time ${ }^{{ }}{ }_{c}$ as the time required for the particle to travel one correlation length along its unperturbed orbit. In quasi-linear thcory this is given by

$$
\therefore \frac{\%}{\mu v} .
$$

We may further define the coherence time ${ }^{-t}$ as the time required for the velocity of the particle to become self-incoherent as it travels along its unperturbed orbit. In quasi-linear theory the orbit is fixed for all realizations of the ensemble so the particle's velocity has no stochastic component and hence never becomes selfincoherent. Therefore obviously in quasi-linear theory $\tau_{c}^{c}=1$ and $\tau_{c}$ is the determining coherence scale. This is not the case in PAF theory.

We have defined two time scales over which the orbit used in calculations becomes unreliable, one for configuration space and one for velocity space. In the same vein we have defined two time scales over which the random force becomes self-incoherent, again one for configuration space and one for velocity space. In Table 2 we list these four time scales for future reference. We require of any theory that 
shorter of $\tau_{c}, \tau_{c}^{\prime}<$ shorter of $\tau_{1}, \tau_{d}$.

In Table 2 we have listed the symbol, name and meaning of each of the four time scales that we have defined here.

We turn now to a comparison of the ratios $\tau_{c} / \tau_{D}, \tau_{c} / t_{i}$ etc. as given by quasi-linea: theory and PAF theory. In the interest of saving space we shall not write down all of the time scale ratios that can be formed although it is a simple matter to do so. We shall rather only explore the consequences of the constraint (61).

In quasi-linear theory the requirement ${ }_{C}<\tau_{D}$ leads to the constraint

$$
\text { , }>\frac{z_{i}}{\mathrm{v}}-\text { for } 2,2, \ldots,
$$

or

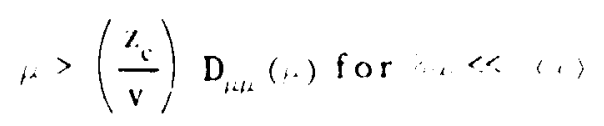

For strong random fields this condition calnnot be met for all (if any) values of $\mu$ but for weak random fields the condition can be met if the power spectrum goes $\mathrm{k}^{-2}$ or steeper for high frequencies (i.e. $\mathrm{D}_{t-\mu}($,$) goes to zero with .. linearly$ or faster).

If, however, one turns to the requirement that ${ }_{c} c \tau_{i}$ one finds for ?

$$
\therefore\left(\frac{a}{v}\right)^{1 / 2}
$$

not much different than before but for , «, , the requirement is 

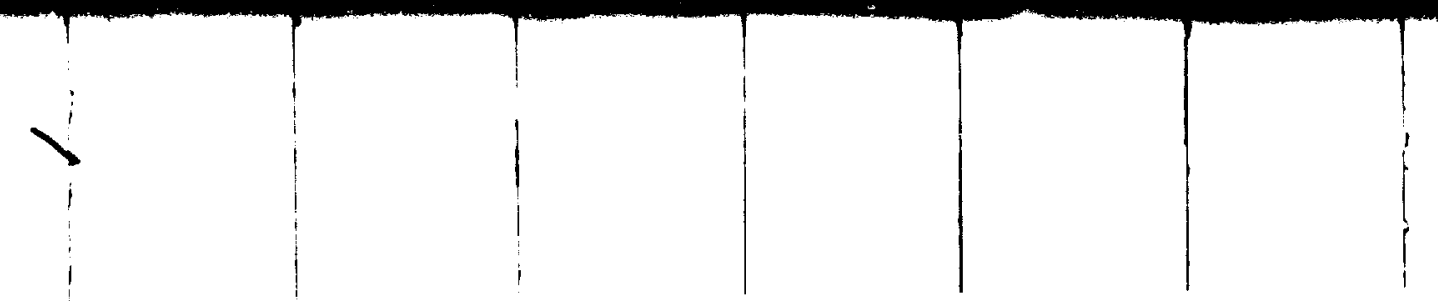

a condition that cannot be met for all values of $\ldots$. We see therefore that quasilinear theory will always have trouble as $\ldots \rightarrow 0$.

The various non-linear theories and PAF theory in particular were introduced to obviate this problem in the vicinity of $i=0$. This is achieved in PAF theory by allowing the fluctuating part of the field to influence the orbit to lowest order. If $o, \ll<$ < ; this inflience may be approximated

$$
z(t) \approx l_{1}^{2}+\cdots,^{2}(1,)^{2} j^{1 / 2} \mathrm{vt}
$$

and hence

$$
\because \frac{{ }^{\prime}}{i,,^{2}+,^{2}\langle\rangle^{2} !^{1 / 2} v} .
$$

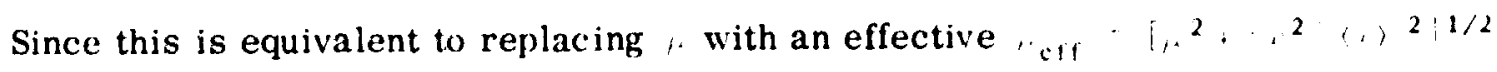
a giance at $(62)$ and $(64)$ indicates that the troubles at $\ldots=0$ are avoided.

However, for the case of strong fluctuating fields, $\therefore\langle<$ this substitution of ". "ff for ". is not a valid approximation. In fact for certain configurations of the fluctuating fields (other than the slab model) the particles can be trapped in the vicinity of the origin for an indefinitely loug time and : can become essentially infinite. In this situation we are saved by the fact that the coherence time $7^{\prime}$ which was infinite in quasilinear theor $y$ is finite in PAF theory and can be the shorter of the correlation/coherence times.

In PAF theory the velocity vector is turned not at the fixed frequency lut at the frequency characteristic of the partially averaged iield

$$
\frac{\mathrm{q}}{\mathrm{mc}}\left|\langle\mathrm{B}\rangle^{2}+\mathrm{B}^{2}\right|^{1 / 2}
$$


During final averaging this frequency will be spread over an amount tia where

$$
\begin{aligned}
& \Delta: \because 8, \text { for } \delta, \geq(1) \\
& \Delta, 2,2,(,) \text { for }, \ll<\text {, }
\end{aligned}
$$

The velocity vector will thus become self-incoherent in a time $\tau_{c}^{\prime}$ given by

$$
\begin{aligned}
& \prime \prime-(A,)^{-1}-b^{-1} \text { for } \therefore \geq(1) \\
&-(1), 2 \text { for } \cdots \ll<(1) .
\end{aligned}
$$

We now find for the various ratios

$$
\begin{aligned}
& \because T_{D}-1 \quad \therefore 2<, \\
& \text { (1) } \mathrm{D}_{1, \mu} \cdot \mu^{2} \\
& \therefore \tau_{11}=\left(\frac{v}{a_{c}}\right)^{1 / 2} \quad \therefore, \therefore \\
& \frac{v}{z_{i}}
\end{aligned}
$$

Since as $v \rightarrow 0 . D_{\mu \mu}$ a $\frac{r^{2}}{(1)}\left(\frac{v}{r+1}\right)^{x}$

where $0 \cdot:$, the only ratio that does not go to zero as $:-0$ is $\because /{ }^{\prime}$ in the strong field case. So we see that even in the case of strong random fields and low rigidity particles there exists an effect that destroys the coherence of the random force at least on the same time scale that the orbit becomes unreliable.

Non-linear theories represent an improvement over quasi-linear thuory for one primary reason: they employ a more realistic description of particle orbits 
in determining the effects of the fluctuating fields on the particles themselves. We have seen that one of the effects of this description is the limiting of the correlation time to a more realistic value such that the resulting calculation remains of marginal validity for a much wider range of parameters. This is true for PAF theory and would presumably be true for any non-linear theory that correctly describes the loss of coherence over'the ensemble of the particle's velocity vector. 


\section{APPENDIX A \\ Conditional Averaging for Multi-Dimensional \\ Gaussian Processes}

Consider a six dimensional vector $\mathrm{x}$ made up of two three dimensional vectors $x_{a}$ and $x_{1}$

$$
\underset{\sim}{x}-\left(x_{i=1}, x_{1}\right)
$$

We wish to find the average value of $x_{b}$ under the condition that $x_{a}={\underset{v}{a y}}_{a}$ If the joint probability density for $x_{,+}$and $x_{b}$ is given as a function of $\underset{\sim}{x}$ as $P(\underset{\sim}{x})$ we have

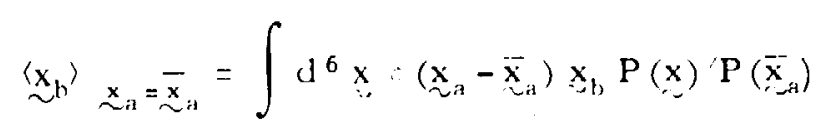

Note that

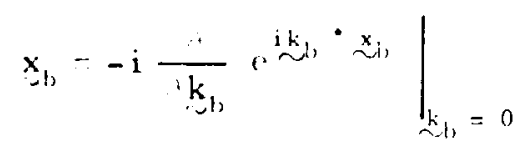

$$
\begin{aligned}
& -\left.i \frac{i}{i k_{-1}} d \stackrel{i k}{\sim} \cdot \underset{\sim}{\sim}\right|_{\mathscr{L}=11}
\end{aligned}
$$

where $\underset{\sim}{\mathrm{k}}$ like $\underset{\sim}{\mathrm{x}}$ is six dimensional. Further, writing the delta function as a Fourier integral

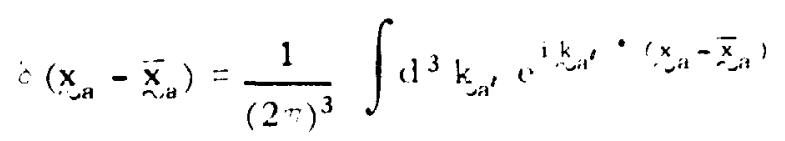

$$
\begin{aligned}
& -\left.\frac{1}{(2 \pi)^{3}} \int d^{3} k_{n a^{\prime}} e^{i k^{\prime} \cdot\left(x_{-}-\bar{x}\right)}\right|_{k_{i}^{\prime}-0}
\end{aligned}
$$


where $\underset{k^{\prime}}{k^{\prime}}$ is also six dimensional, we may insert these expressions into (A2) to obtain

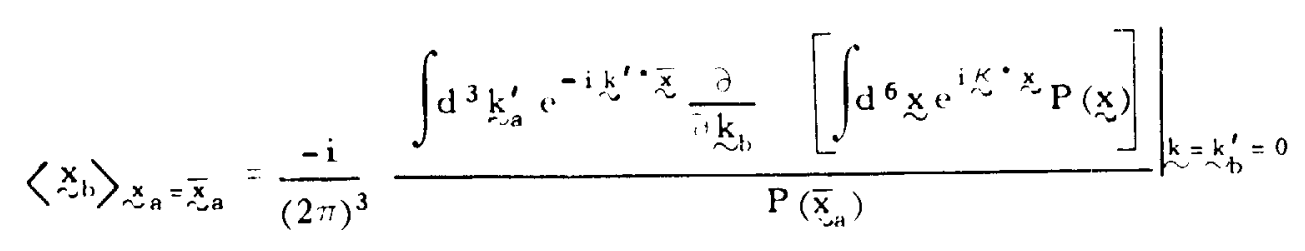

where $\underset{\sim}{\kappa}=\underset{\sim}{\mathrm{k}}+\underset{\sim}{\mathrm{k}^{\prime}}$.

The quantity in square braclets is the characteristic function of the distribution function $P(x)$

$$
\text { Characteristic fn. } \tilde{\mathrm{P}}(\kappa)-\langle c \stackrel{i}{i} \stackrel{x}{-}\rangle
$$

If $x$ is a Gaussian process the distribution $P(x)$ is a multivariate Gaussian ${ }^{31}$

$$
P(x)-(2 \cdots)^{-3}|M|^{-1} \exp \left(-\frac{1}{2} x \cdot M^{-1} \cdot x\right)
$$

Where $\underset{\because}{M^{-1}}$ is the inverse of the covariance matrix $\underset{\sim}{M}$. i.e.. $M_{i j}\left\langle x_{i} x_{j}\right\rangle$ an obviously symmetric matrix. In complete analogy with a one dimensional Gaussian distribution it is straightforward to show that

$$
\breve{P}(t)-\exp \left(-\frac{1}{2} \because \cdot M \cdot k\right) .
$$

If we now break $\underset{\approx}{M}$ up into the three by three matrices $\underset{\approx}{S}=\left\langle x_{a} x_{r a}\right\rangle=\left\langle x_{1}, x_{b}\right\rangle$ and $\underset{\approx}{\mathrm{C}}=\left\langle\mathrm{x}_{\mathrm{a}} \mathrm{x}_{\mathrm{b}}\right\rangle, \mathrm{C}^{\mathrm{T}}=\left\langle\mathrm{x}_{\mathrm{H}}, \mathrm{x}_{\mathrm{i}}\right\rangle$ where we have assumed that the marginal distributions for ${\underset{\sim a}{\mathrm{x}}}_{\mathrm{a}}$ and $\underset{\sim}{\mathrm{x}}$, are identical we have

$$
\stackrel{M}{\approx}\left(\begin{array}{cc}
S & C \\
\ddots & \approx \\
C^{\top} & S
\end{array}\right)
$$

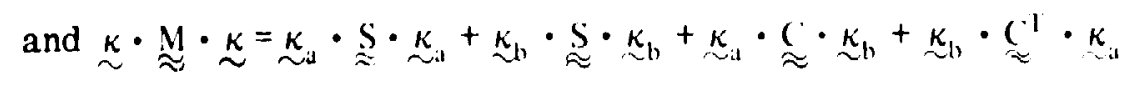




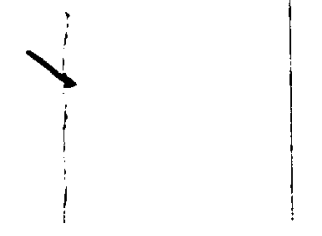

APPENDIX B

The Gyrotropy of $\langle\mathrm{f}\rangle$

Consider the equation for $\langle f\rangle$

$$
\frac{\partial\langle f\rangle}{\partial t}+\langle i\rangle \frac{i\langle f\rangle}{\partial f}=D(t)\langle f\rangle
$$

where $\mathrm{D}(t)$ represents the complete collision operator. If $\mathrm{D}(\neq)$ were not a function of the gyro-phase $i$, we could average this equation over $;$ and remove it from the problem but the $\neq$, dependence of $D$ and $\langle f\rangle$ will average together to produce an unknown phase correlation.

Let us break $\langle f\rangle$ into a gyrotropic part $\langle f\rangle_{0}$ and $a \neq$ dependent part $\langle f\rangle_{1}$. If we indicate the average over gyrophase of a quantity by placing a bas over it, we further specify that $\overline{\langle\mathrm{f}}\rangle=\langle\mathrm{f}\rangle_{0}$ and $\left.\overline{\langle\mathrm{f}}\right\rangle_{1}=0$.

Inserting in (B1) and averaging over $\ddagger$ gives

$$
\frac{i\langle f\rangle_{0}}{i t}-\bar{D}\langle f\rangle_{0}+\overline{D\langle f\rangle_{1}} .
$$

Subtracting (B2) from (B1) gives

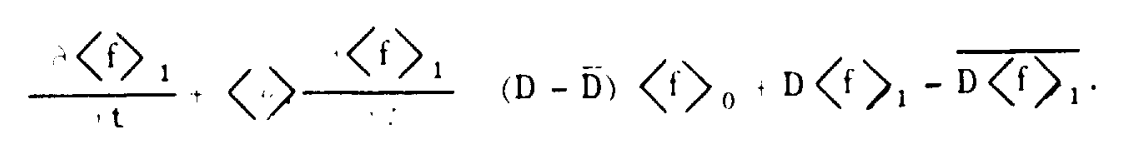

We now shall est wat if cerms in (B3) as follow's

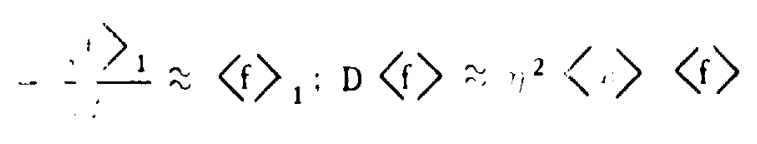

to obtain

$$
\frac{\langle f\rangle_{1}}{t}+\langle\rangle\langle f\rangle_{1} \because r_{i}^{2}\langle f\rangle\langle f\rangle_{0}
$$




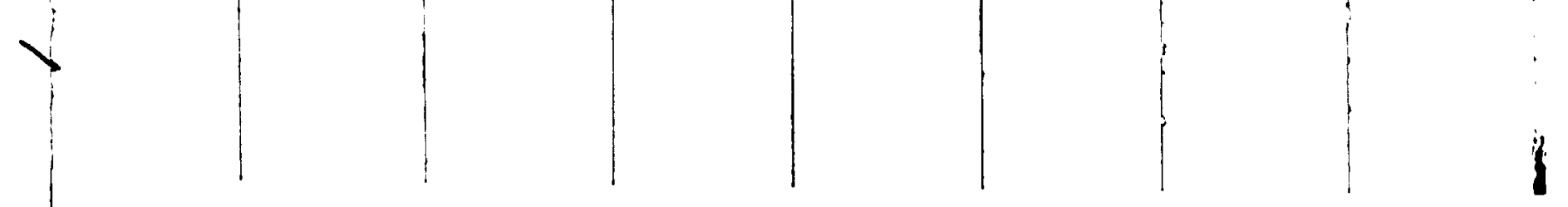

where we now assume $\eta^{2}<1$ and we neglect $\eta^{2}\langle\omega\rangle\langle f\rangle_{1}$ compared to $\langle\omega\rangle\langle f\rangle_{1}$. Integrating $(\mathrm{B} 4)$ with $\langle\mathrm{f}\rangle_{1}(t=0)=0$ gives

$$
\begin{aligned}
\langle\mathrm{f}\rangle_{1} \approx \eta^{2}\langle\omega\rangle & \int_{0}^{\mathrm{t}} \exp [-\langle\omega\rangle(\mathrm{t}-\tau)]\langle\mathrm{f}\rangle_{0}(\tau) \mathrm{d} \tau \\
& \approx \eta^{2}\langle\mathrm{f}\rangle_{0}+O\left(\eta^{4}\right)
\end{aligned}
$$

for $t \gg\langle\omega\rangle-$ ?

We see from (B5) that $\langle\mathrm{f}\rangle_{1}$ is $\mathrm{O}\left(\eta^{2}\right)$ if $\langle\mathrm{f}\rangle_{0}$ is $\mathrm{O}(1)$; therefore the second term on the right hand side of $(\mathrm{B} 2)$ is of $O\left(r^{4}\right)$ and may be neglected. 
Table 1

The Parallel Spatial Diffusion Coefficients as Calculated

Using $\mathrm{D}_{\mu \mu}^{\mathrm{PA}}$ by the Legendre and Inversion Methods

\begin{tabular}{|c|c|c|}
\hline Case & $\kappa_{\mathrm{H}}^{\mathrm{L}}\left\langle\omega / \mathrm{v}^{2}\right.$ & $\kappa_{\|}^{\mathrm{J}}\left\langle u \mathrm{v}^{2}\right.$ \\
\hline$\epsilon=1, \gamma_{i}=.05,1 \mathrm{slab}$ & 456 & 622 \\
$\epsilon=1, \gamma=.1,1 \mathrm{slab}$ & 115 & 137 \\
$\epsilon=1, \gamma_{i}=.3,1$ slab & 13.06 & 13.48 \\
$\epsilon=1, \eta=.1$, oblique slab & 162 & 367 \\
\hline
\end{tabular}




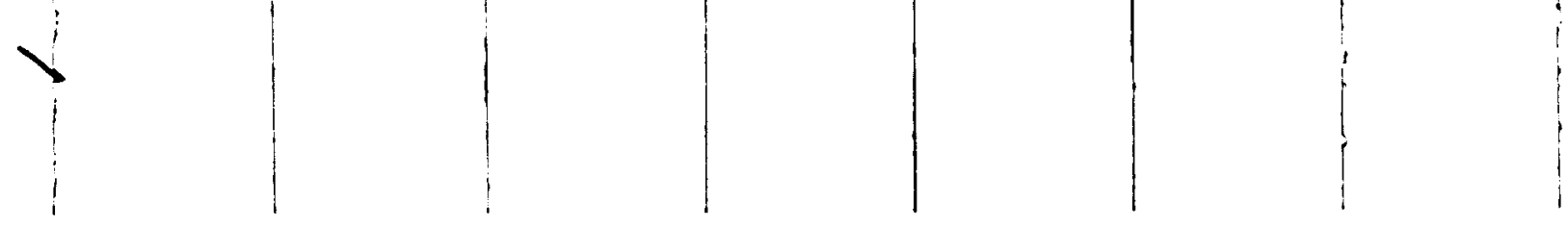

Table 2

Time Scales

\begin{tabular}{|c|c|c|}
\hline Symbol & Name & \multicolumn{1}{c|}{ Meaning } \\
\hline${ }^{\prime}$ D & Deflection time & $\begin{array}{l}\text { Time required for orbit to become } \\
\text { unreliable in velocity space }\end{array}$ \\
& Deviation time & $\begin{array}{l}\text { Time required for orbit to become } \\
\text { unreliable in configuration space }\end{array}$ \\
& Correlation time & $\begin{array}{l}\text { Time required for magnetic field } \\
\text { to become self-incoherent }\end{array}$ \\
& Coherence time & $\begin{array}{l}\text { Time required for velocity to be- } \\
\text { come seli-incoherent }\end{array}$ \\
\hline
\end{tabular}




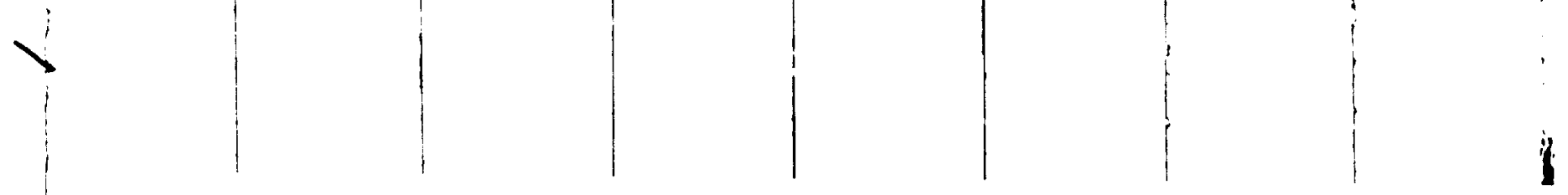

\section{Figure Captions}

Figure 1. The diffusion coefficient $\mathrm{D}_{l i \mu}$ for the perpendicular slab model when $\epsilon=\eta_{\mathrm{c}} \mathrm{m},\langle\mathrm{s}\rangle / \mathrm{p}=1$ and $r=\left\langle\mathrm{B}^{2}\right\rangle{ }^{1 / 2} /\langle\mathrm{B}\rangle=.05$.

Figure 2. $D_{\mu \mu}$ vs. $\mu$ for the perpendicular slab model when $c_{2}=1$ and $\gamma_{i}=.1$

Figure 3. $\mathrm{D}_{\mu \mu}$ vs. $/$ for the perpendicular slab model when $\epsilon=1$ and $;=.3$

Figure 4. The $p_{x}, p_{y}$ plane showing regions where particle exits toward $+z$ $(\langle\mu\rangle\rangle 0$,$) , particle exits toward -z(\langle\mu\rangle,\langle 0)$, and where mirroring can possibly occur (indicated by "M?").

Figure 5. $\mathrm{D}_{\mu \mu}$ vs. $\mu$ for the oblique slab model when $=1$ and $;=.1$ 


\section{References}

1. J. R. Jokipii, Astrophys. J. 146, 480 (1966).

2. E. C. Roelof, Ph.D. dissertation, University of California, Berkeley, 1966.

3. D. E. Hall and P. A. Sturrock, Phys. Fluids $\underline{10}, 2620$ (1967).

4. A. A. Vedenov, E. P. Velikhov, and R. Z. Sagdeev, Nucl. Fusion 1, 82 (1961); Nucl. Fusion Suppl. 2, 465 (1962); W. E. Drummond and D. Pines, Nucl. Fusion Suppl. $\underline{3}, 1049$ (1962).

5. J. R. Jokipii, Rev. Geophys. and Space Phys. $\underline{0}, 27$ (1971); Astrophys. J. $\underline{172}$, 319 (1972).

6. K. Hasselman and G. Wibberenz, Zs. f. Geophys. $\underline{34}, 353$ (1968).

7. C. E. Newman, J. Math. Phys. 14, 502 (1973).

8. R. Kulsrud and W. P. Pearce, Astrophys. J. $\underline{156}, 445$ (1969).

9. A. N. Kaufman, Phys. Fluids $\underline{11}, 326$ (1968).

10. A. J. Klimas and G. Sandri, Astrophy's. J. 169, 41 (1971).

11. T. B. Kaiser, F. C. Jones, and T. J. Birmingham, A strophys. J. 180, 239 (1973).

12. J. A. Earl, Astrophys. J. $\underline{188}, 379$ (1974).

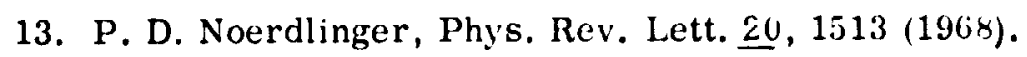

14. T. H. Dupree, Phys. Fluids 9, 1773 (1966); Phys. Fluids 10, 1049 (1967); J. Weinstock, Phys. Fluids. 11, 1977 (1968).

15. M. L. Goldstein, Astrophys. J. 204, 900 (1976).

16. A. J. Owens, Astrophys. J. $\underline{191}, 235$ (1974); H. J. Völk, Astrophys. and Space Sci. 25,471 (1973). 

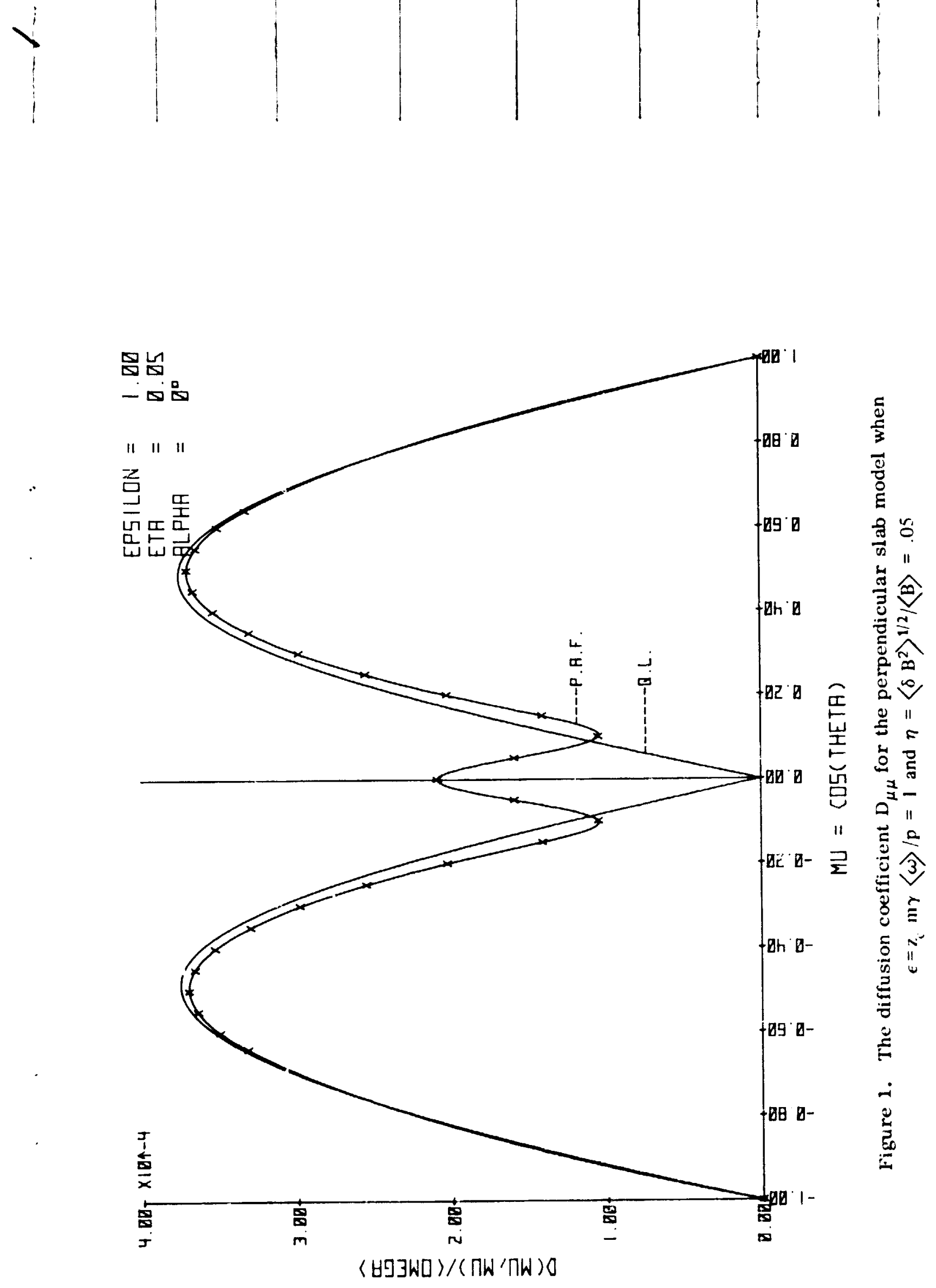

PKELLUING PAGE BLANK NOS SIIT: 


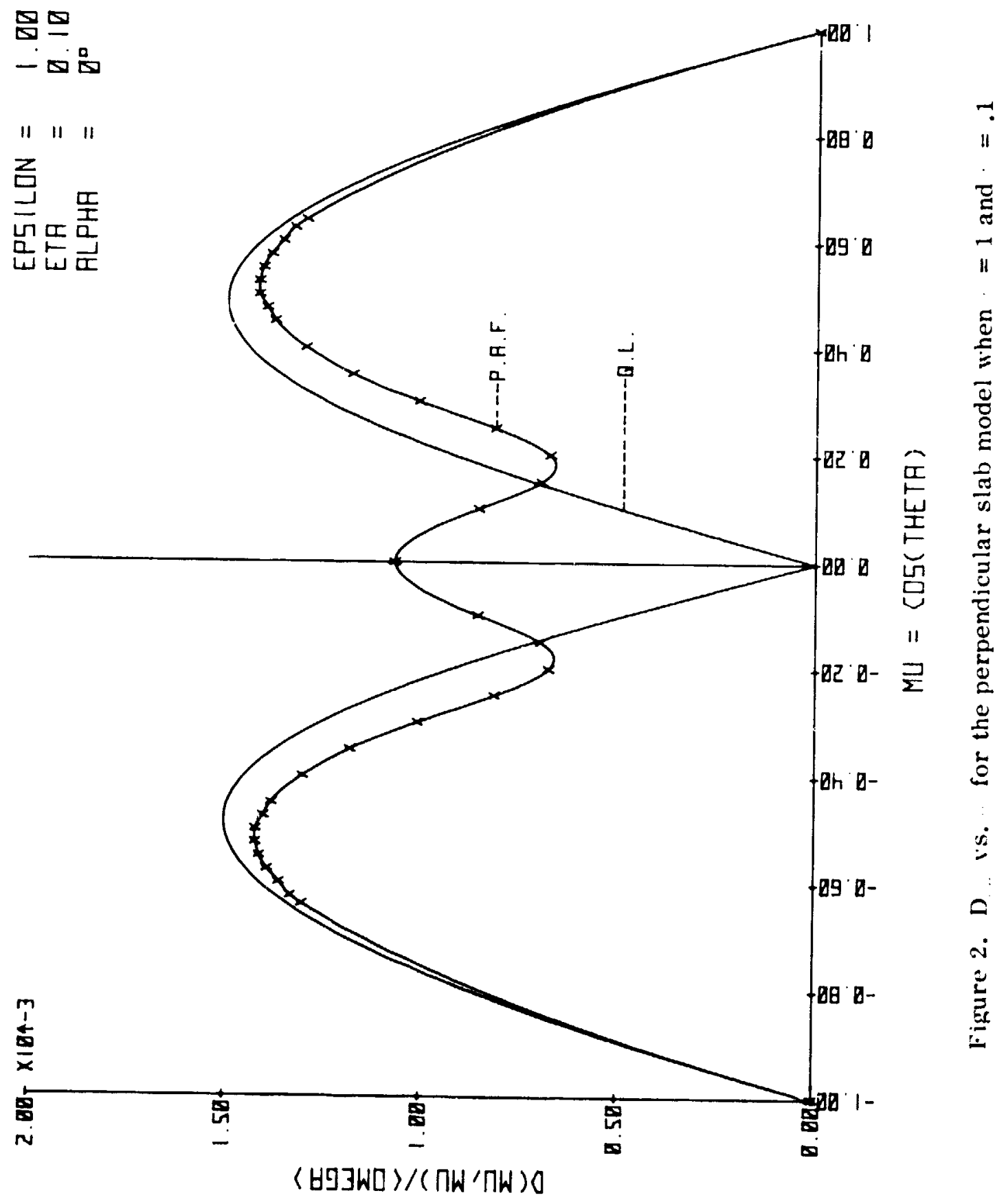




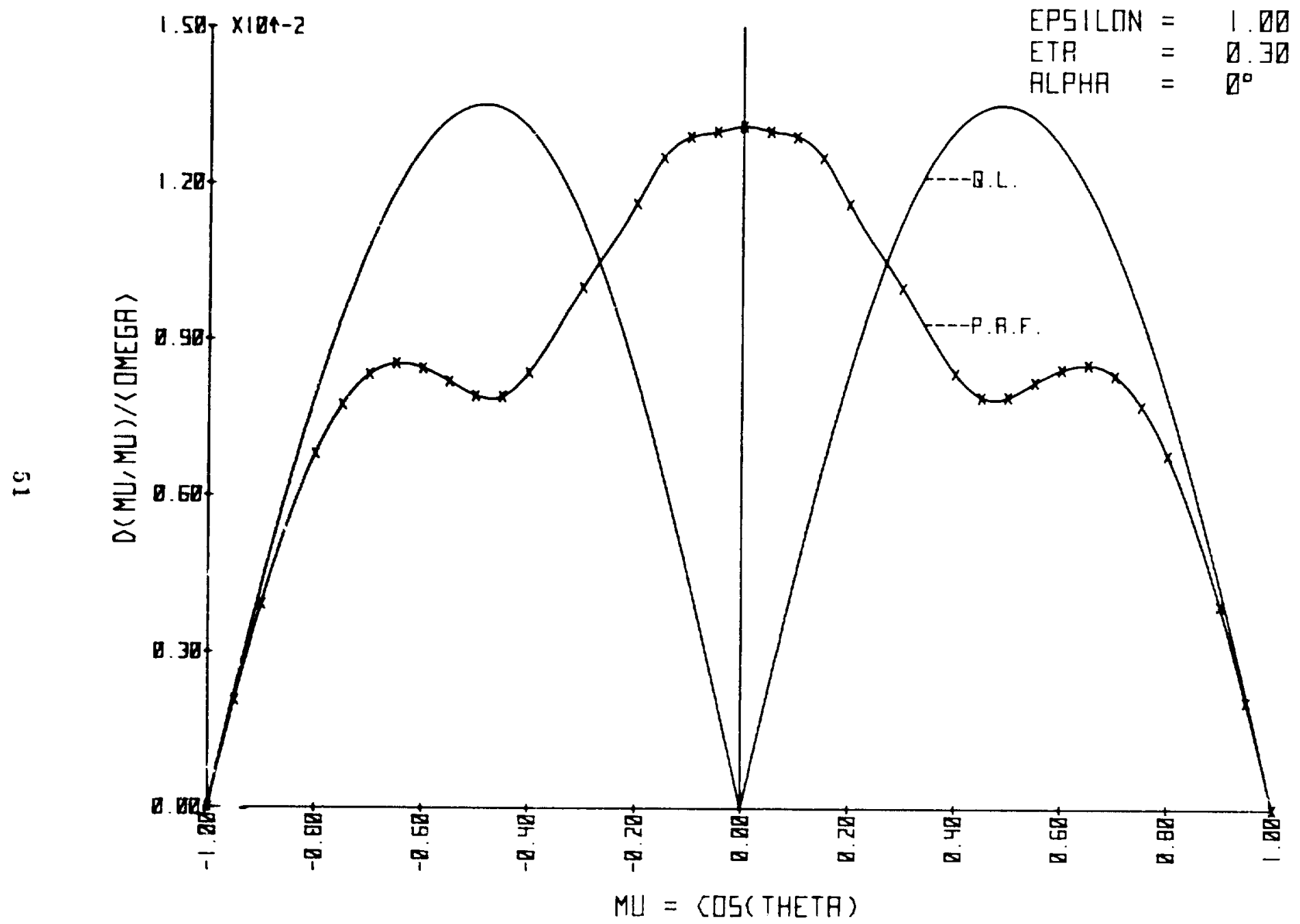

Figure 3. D is. for the perpendicular slab model when $=1$ and $=.3$ 


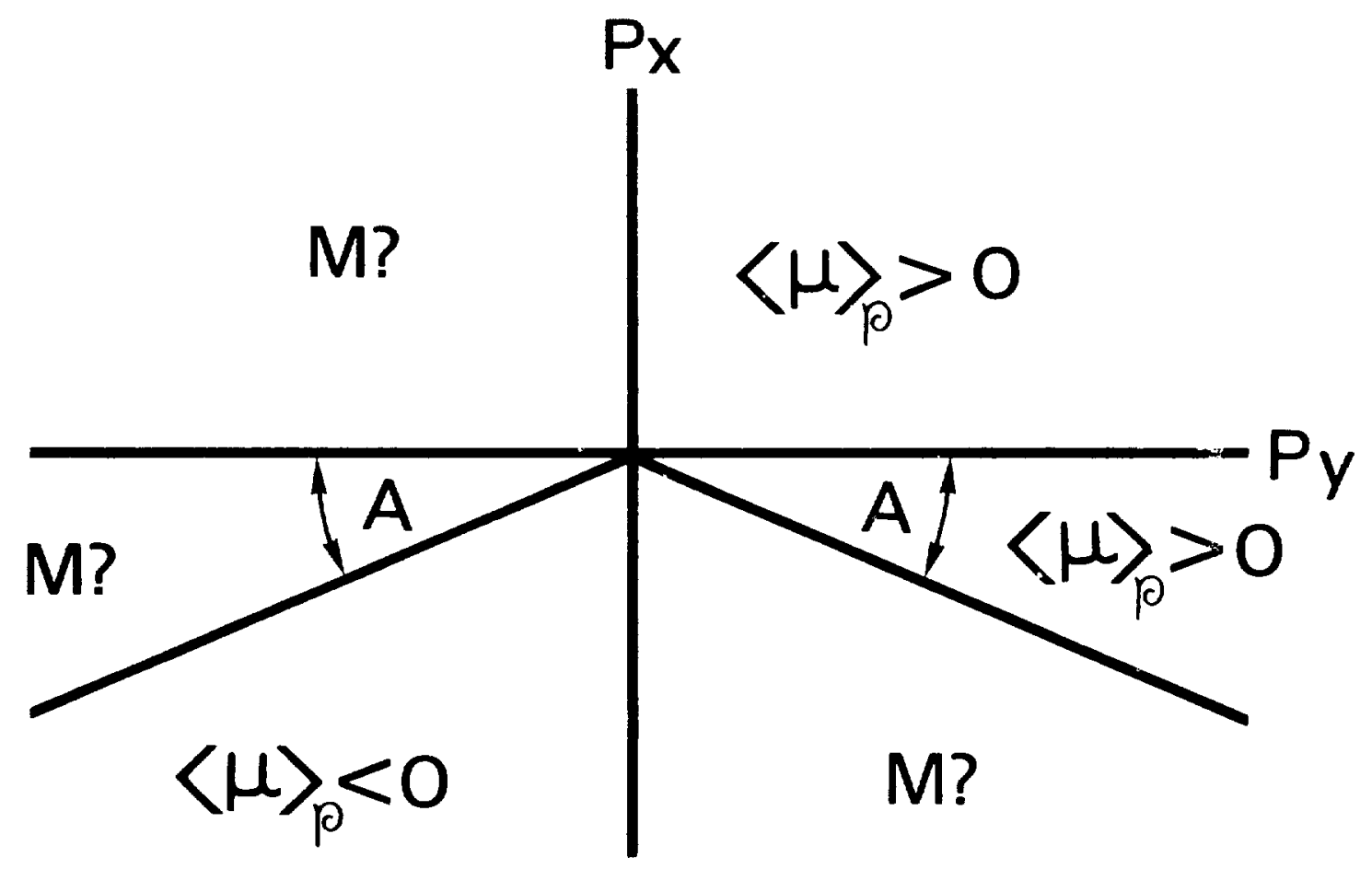

Figure 4. The $p_{1}, p_{y}$ plane showing regions where particle exits toward $+z(\langle .1\rangle, 0)$, particle exits toward $-z(\ldots,-0)$, and where mirroring can possibly occur (indicated by "M?"). 

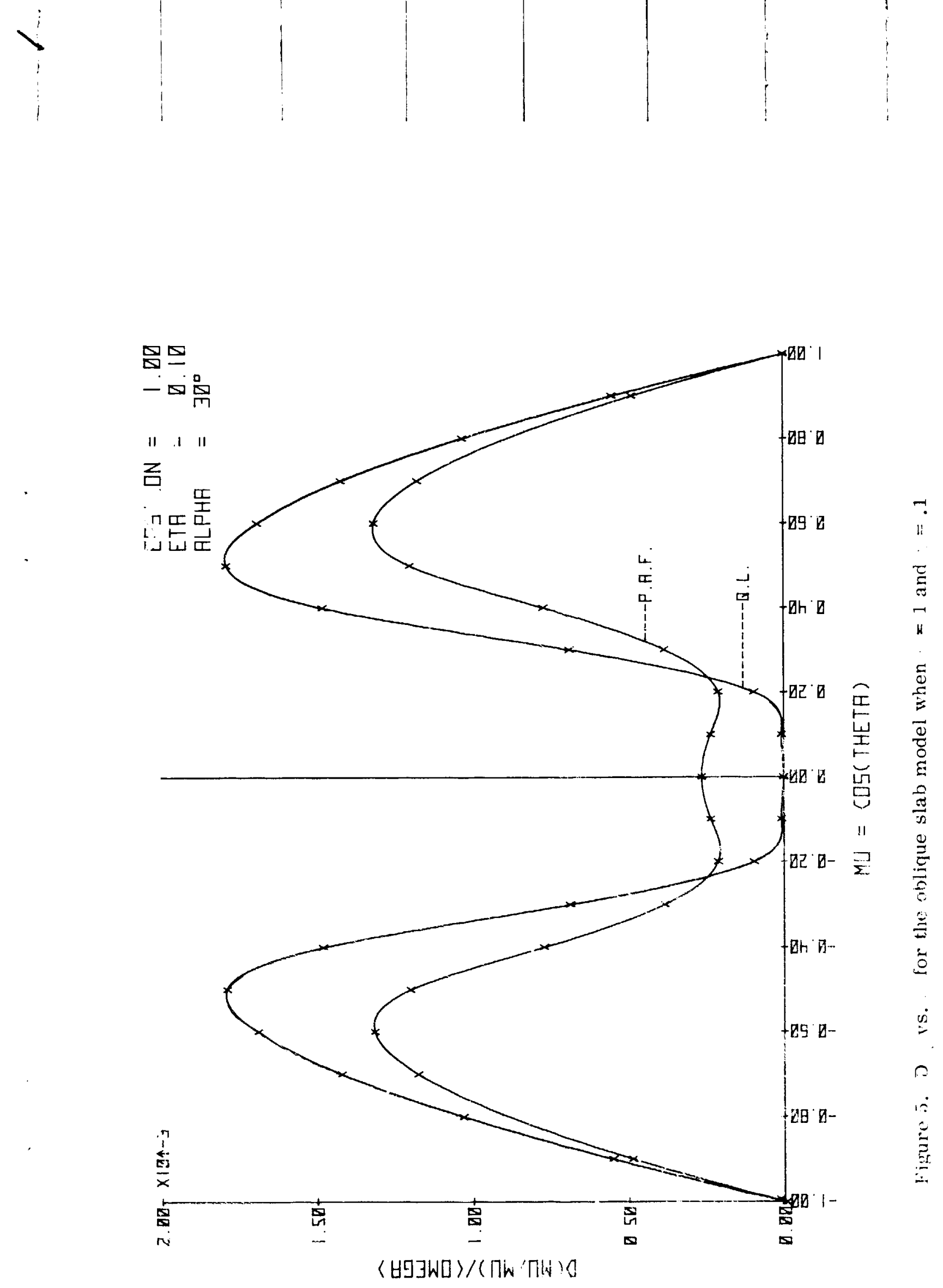